\title{
Searches for Continuous Gravitational Waves from 15 Supernova Remnants and Fomalhaut b with Advanced LIGO*
}

B. P. Abbott ${ }^{1}$, R. Abbott ${ }^{1}$, T. D. Abbott ${ }^{2}$, S. Abraham ${ }^{3}$, F. Acernese ${ }^{4,5}$, K. Ackley ${ }^{6}$, C. Adams $^{7}$, R. X. Adhikari ${ }^{1}$, V. B. Adya ${ }^{8,9}$, C. Affeldt ${ }^{8,9}$, M. Agathos ${ }^{10}$, K. Agatsuma ${ }^{11}$, N. Aggarwal ${ }^{12}$, O. D. Aguiar ${ }^{13}$, L. Aiello ${ }^{14,15}$, A. Ain ${ }^{3}$, P. Ajith ${ }^{16}$, G. Allen ${ }^{17}$ A. Allocca ${ }^{18,19}$, M. A. Aloy ${ }^{20}$, P. A. Altin ${ }^{21}$, A. Amato ${ }^{22}$, A. Ananyeva ${ }^{1}$, S. B. Anderson ${ }^{1}$, W. G. Anderson ${ }^{23}$, S. V. Angelova ${ }^{24}$, S. Antier ${ }^{25}$, S. Appert ${ }^{1}$, K. Arai $^{1}$, M. C. Araya ${ }^{1}$, J. S. Areeda ${ }^{26}$, M. Arène ${ }^{27}$, N. Arnaud ${ }^{25,28}$, K. G. Arun ${ }^{29}$, S. Ascenzi $^{30,31}$,

G. Ashton ${ }^{6}$, S. M. Aston ${ }^{7}$, P. Astone ${ }^{32}$, F. Aubin ${ }^{33}$, P. Aufmuth ${ }^{9}$, K. AultONeal ${ }^{34}$, C. Austin ${ }^{2}$, V. Avendano ${ }^{35}$, A. Avila-Alvarez ${ }^{26}$, S. Babak ${ }^{27,36}$, P. Bacon ${ }^{27}$, F. Badaracco ${ }^{14,15}$, M. K. M. Bader ${ }^{37}$, S. Bae ${ }^{38}$, P. T. Baker ${ }^{39}$, F. Baldaccini ${ }^{40,41}$, G. Ballardin ${ }^{28}$, S. W. Ballmer ${ }^{42}$, S. Banagiri ${ }^{43}$, J. C. Barayoga ${ }^{1}$, S. E. Barclay ${ }^{44}$, B. C. Barish ${ }^{1}$, D. Barker ${ }^{45}$, K. Barkett ${ }^{46}$, S. Barnum ${ }^{12}$, F. Barone ${ }^{4,5}$, B. Barr ${ }^{44}$, L. Barsotti ${ }^{12}$, M. Barsuglia ${ }^{27}$, D. Barta ${ }^{47}$, J. Bartlett ${ }^{45}$, I. Bartos ${ }^{48}$, R. Bassiri ${ }^{49}$, A. Basti ${ }^{18,19}$, M. Bawaj ${ }^{41,50}$, J. C. Bayley ${ }^{44}$, M. Bazzan ${ }^{51,52}$, B. Bécsy ${ }^{53}$, M. Bejger ${ }^{27,54}$, I. Belahcene ${ }^{25}$, A. S. Bell ${ }^{44}$, D. Beniwal ${ }^{55}$, B. K. Berger ${ }^{49}$, G. Bergmann ${ }^{8,9}$, S. Bernuzzi ${ }^{56,57}$, J. J. Bero ${ }^{58}$, C. P. L. Berry ${ }^{59}$, D. Bersanetti ${ }^{60}$, A. Bertolini ${ }^{37}$, J. Betzwieser ${ }^{7}$, R. Bhandare ${ }^{61}$, J. Bidler $^{26}$, I. A. Bilenko ${ }^{62}$, S. A. Bilgili ${ }^{39}$, G. Billingsley ${ }^{1}$, J. Birch ${ }^{7}$, R. Birney ${ }^{24}$, O. Birnholtz ${ }^{58}$, S. Biscans ${ }^{1,12}$, S. Biscoveanu ${ }^{6}$, A. Bisht ${ }^{9}$, M. Bitossi ${ }^{19,28}$, M. A. Bizouard ${ }^{25}$, J. K. Blackburn ${ }^{1}$, C. D. Blair ${ }^{7}$, D. G. Blair ${ }^{63}$, R. M. Blair ${ }^{45}$, S. Bloemen ${ }^{64}$, N. Bode ${ }^{8,9}$, M. Boer ${ }^{65}$, Y. Boetzel ${ }^{66}$, G. Bogaert ${ }^{65}$, F. Bondu ${ }^{67}$, E. Bonilla ${ }^{49}$, R. Bonnand ${ }^{33}$, P. Booker ${ }^{8,9}$, B. A. Boom ${ }^{37}$, C. D. Booth ${ }^{68}$, R. Bork ${ }^{1}$, V. Boschi ${ }^{28}$, S. Bose ${ }^{3,69}$, K. Bossie ${ }^{7}$, V. Bossilkov ${ }^{63}$, J. Bosveld ${ }^{63}$, Y. Bouffanais ${ }^{27}$, A. Bozzi ${ }^{28}$, C. Bradaschia ${ }^{19}$, P. R. Brady ${ }^{23}$, A. Bramley ${ }^{7}$, M. Branchesi ${ }^{14,15}$, J. E. Brau ${ }^{70}$, T. Briant ${ }^{71}$, J. H. Briggs ${ }^{44}$, F. Brighenti ${ }^{72,73}$, A. Brillet ${ }^{65}$, M. Brinkmann ${ }^{8,9}$, V. Brisson ${ }^{25,176}$, P. Brockill ${ }^{23}$, A. F. Brooks ${ }^{1}$, D. D. Brown ${ }^{55}$, S. Brunett ${ }^{1}$, A. Buikema ${ }^{12}$, T. Bulik ${ }^{74}$, H. J. Bulten ${ }^{37,75}$, A. Buonanno ${ }^{36,76}$, D. Buskulic ${ }^{33}$, C. Buy ${ }^{27}$, R. L. Byer ${ }^{49}$, M. Cabero ${ }^{8,9}$, L. Cadonati ${ }^{77}$, G. Cagnoli ${ }^{22,78}$, C. Cahillane ${ }^{1}$, J. Calderón Bustillo ${ }^{6}$, T. A. Callister ${ }^{1}$, E. Calloni ${ }^{5,79}$, J. B. Camp ${ }^{80}$, W. A. Campbell ${ }^{6}$, K. C. Cannon ${ }^{81}$, H. Cao ${ }^{55}$, J. Cao ${ }^{82}$, E. Capocasa $^{27}$, F. Carbognani ${ }^{28}$, S. Caride ${ }^{83}$, M. F. Carney ${ }^{59}$, G. Carullo ${ }^{18}$, J. Casanueva Diaz ${ }^{19}$, C. Casentini ${ }^{30,31}$,

S. Caudill ${ }^{37}$, M. Cavaglià ${ }^{84}$, F. Cavalier ${ }^{25}$, R. Cavalieri ${ }^{28}$, G. Cella ${ }^{19}$, P. Cerdá-Durán ${ }^{20}$, G. Cerretani ${ }^{18,19}$, E. Cesarini ${ }^{31,85}$,', O. Chaibi ${ }^{65}$, K. Chakravarti ${ }^{3}$, S. J. Chamberlin ${ }^{86}$, M. Chan ${ }^{44}$, S. Chao ${ }^{87}$, P. Charlton ${ }^{88}$, E. A. Chase ${ }^{59}$, E. Chassande-Mottin ${ }^{27}$, D. Chatterjee ${ }^{23}$, M. Chaturvedi ${ }^{61}$, B. D. Cheeseboro ${ }^{39}$, H. Y. Chen ${ }^{89}$, X. Chen ${ }^{63}$, Y. Chen ${ }^{46}$, H.-P. Cheng ${ }^{48}$, C. K. Cheong ${ }^{90}$, H. Y. Chia ${ }^{48}$, A. Chincarini ${ }^{60}$, A. Chiummo ${ }^{28}$, G. Cho $^{91}$, H. S. Cho ${ }^{92}$, M. Cho ${ }^{76}$, N. Christensen ${ }^{65,93}$, Q. Chu ${ }^{63}$, S. Chua ${ }^{71}$, K. W. Chung ${ }^{90}$, S. Chung ${ }^{63}$, G. Ciani ${ }^{51,52}$, A. A. Ciobanu ${ }^{55}$, R. Ciolfi ${ }^{94,95}$, F. Cipriano ${ }^{65}$, A. Cirone ${ }^{60,96}$, F. Clara ${ }^{45}$, J. A. Clark ${ }^{77}$, P. Clearwater ${ }^{97}$, F. Cleva ${ }^{65}$, C. Cocchieri ${ }^{84}$, E. Coccia ${ }^{14,15}$, P.-F. Cohadon ${ }^{71}$, D. Cohen ${ }^{25}$, R. Colgan ${ }^{98}$, M. Colleoni ${ }^{99}$, C. G. Collette ${ }^{100}$, C. Collins ${ }^{11}$, L. R. Cominsky ${ }^{101}$, M. Constancio, Jr. ${ }^{13}$, L. Conti ${ }^{52}$, S. J. Cooper ${ }^{11}$, P. Corban ${ }^{7}$, T. R. Corbitt ${ }^{2}$, I. Cordero-Carrión ${ }^{12}$, K. R. Corley ${ }^{98}$, N. Cornish ${ }^{53}$, A. Corsi ${ }^{83}$, S. Cortese ${ }^{28}$, C. A. Costa ${ }^{13}$, R. Cotesta ${ }^{36}$, M. W. Coughlin ${ }^{1}$,

S. B. Coughlin ${ }^{59,68}$, J.-P. Coulon ${ }^{65}$, S. T. Countryman ${ }^{98}$, P. Couvares ${ }^{1}$, P. B. Covas ${ }^{99}$, E. E. Cowan ${ }^{77}$, D. M. Coward ${ }^{63}$,

M. J. Cowart ${ }^{7}$, D. C. Coyne $^{1}$, R. Coyne ${ }^{103}$, J. D. E. Creighton ${ }^{23}$, T. D. Creighton ${ }^{104}$, J. Cripe ${ }^{2}$, M. Croquette ${ }^{71}$, S. G. Crowder ${ }^{105}$, T. J. Cullen ${ }^{2}$, A. Cumming ${ }^{44}$, L. Cunningham ${ }^{44}$, E. Cuoco ${ }^{28}$, T. Dal Canton ${ }^{80}$, G. Dálya ${ }^{106}$, S. L. Danilishin ${ }^{8,9}$, S. D’Antonio ${ }^{31}$, K. Danzmann ${ }^{8,9}$, A. Dasgupta ${ }^{107}$, C. F. Da Silva Costa ${ }^{48}$, L. E. H. Datrier ${ }^{44}$, V. Dattilo ${ }^{28}$, I. Dave ${ }^{61}$, M. Davier ${ }^{25}$, D. Davis ${ }^{42}$,

E. J. Daw ${ }^{108}$, D. DeBra ${ }^{49}$, M. Deenadayalan ${ }^{3}$, J. Degallaix ${ }^{22}$, M. De Laurentis ${ }^{5,79}$, S. Deléglise ${ }^{71}$, W. Del Pozzo ${ }^{18,19}$, L. M. DeMarchi ${ }^{59}$, N. Demos ${ }^{12}$, T. Dent ${ }^{8,9}$, M. Denys ${ }^{74}$, R. De Pietri ${ }^{57,109}$, J. Derby ${ }^{26}$, R. De Rosa ${ }^{5,79}$, C. De Rossi ${ }^{22,28}$, R. DeSalvo ${ }^{110}$, O. de Varona ${ }^{8,9}$, S. Dhurandhar ${ }^{3}$, M. C. Díaz ${ }^{104}$, T. Dietrich ${ }^{37}$, L. Di Fiore ${ }^{5}$, M. Di Giovanni9 ${ }^{95,111}$,

T. Di Girolamo 5,79 , A. Di Lieto ${ }^{18,19}$, B. Ding ${ }^{100}$, S. Di Pace ${ }^{32,112}$, I. Di Palma ${ }^{32,112}$, F. Di Renzo ${ }^{18,19}$, A. Dmitriev ${ }^{11}$, Z. Doctor ${ }^{89}$, F. Donovan ${ }^{12}$, K. L. Dooley ${ }^{68,84}$, S. Doravari ${ }^{8,9}$, I. Dorrington ${ }^{68}$, T. P. Downes ${ }^{23}$, M. Drago ${ }^{14,15}$, J. C. Driggers ${ }^{45}$, Z. Du ${ }^{82}$, J.-G. Ducoin ${ }^{25}$, P. Dupej ${ }^{44}$, S. E. Dwyer ${ }^{45}$, P. J. Easter ${ }^{6}$, T. B. Edo $^{108}$, M. C. Edwards ${ }^{93}$, A. Effler ${ }^{7}$, P. Ehrens ${ }^{1}$, J. Eichholz ${ }^{1}$, S. S. Eikenberry ${ }^{48}$, M. Eisenmann ${ }^{33}$, R. A. Eisenstein ${ }^{12}$, R. C. Essick ${ }^{89}$, H. Estelles ${ }^{99}$, D. Estevez ${ }^{33}$, Z. B. Etienne ${ }^{39}$, T. Etzel ${ }^{1}$, M. Evans ${ }^{12}$, T. M. Evans ${ }^{7}$, V. Fafone ${ }^{14,30,31}$, H. Fair ${ }^{42}$, S. Fairhurst ${ }^{68}$, X. Fan $^{82}$, S. Farinon ${ }^{60}$, B. Farr ${ }^{70}$, W. M. Farr ${ }^{11}$,

E. J. Fauchon-Jones ${ }^{68}$, M. Favata ${ }^{35}$, M. Fays ${ }^{108}$, M. Fazio ${ }^{113}$, C. Fee ${ }^{114}$, J. Feicht ${ }^{1}$, M. M. Fejer ${ }^{49}$, F. Feng ${ }^{27}$,

A. Fernandez-Galiana ${ }^{12}$, I. Ferrante ${ }^{18,19}$, E. C. Ferreira ${ }^{13}$, T. A. Ferreira ${ }^{13}$, F. Ferrini2 ${ }^{28}$, F. Fidecaro ${ }^{18,19}$, I. Fiori ${ }^{28}$, D. Fiorucci ${ }^{27}$, M. Fishbach ${ }^{89}$, R. P. Fisher ${ }^{42,115}$, J. M. Fishner ${ }^{12}$, M. Fitz-Axen ${ }^{43}$, R. Flaminio ${ }^{33,116}$, M. Fletcher ${ }^{44}$, E. Flynn ${ }^{26}$, H. Fong ${ }^{117}$, J. A. Font ${ }^{20,118}$, P. W. F. Forsyth ${ }^{21}$, J.-D. Fournier ${ }^{65}$, S. Frasca ${ }^{32,112}$, F. Frasconi ${ }^{19}$, Z. Frei ${ }^{106}$, A. Freise ${ }^{11}$, R. Frey ${ }^{70}$, V. Frey ${ }^{25}$, P. Fritschel ${ }^{12}$, V. V. Frolov ${ }^{7}$, P. Fulda ${ }^{48}$, M. Fyffe ${ }^{7}$, H. A. Gabbard ${ }^{44}$, B. U. Gadre ${ }^{3}$, S. M. Gaebel ${ }^{11}$, J. R. Gair ${ }^{19}$, L. Gammaitoni ${ }^{40}$, M. R. Ganija ${ }^{55}$, S. G. Gaonkar ${ }^{3}$, A. Garcia ${ }^{26}$, C. García-Quirós ${ }^{99}$, F. Garufi ${ }^{5,79}$, B. Gateley ${ }^{45}$, S. Gaudio ${ }^{34}$, G. Gaur ${ }^{120}$, V. Gayathri ${ }^{121}$, G. Gemme ${ }^{60}$, E. Genin ${ }^{28}$, A. Gennai ${ }^{19}$, D. George ${ }^{17}$, J. George ${ }^{61}$, L. Gergely ${ }^{122}$, V. Germain ${ }^{33}$, S. Ghonge ${ }^{77}$, Abhirup Ghosh ${ }^{16}$, Archisman Ghosh ${ }^{37}$, S. Ghosh ${ }^{23}$, B. Giacomazzo ${ }^{95,111}$, J. A. Giaime ${ }^{2,7}$, K. D. Giardina ${ }^{7}$, A. Giazotto ${ }^{19,177}$, K. Gill ${ }^{34}$, G. Giordano ${ }^{4,5}$, L. Glover ${ }^{110}$, P. Godwin ${ }^{86}$, E. Goetz ${ }^{45}$, R. Goetz ${ }^{48}$, B. Goncharov ${ }^{6}$, G. González ${ }^{2}$,

J. M. Gonzalez Castro ${ }^{18,19}$, A. Gopakumar ${ }^{123}$, M. L. Gorodetsky ${ }^{62}$, S. E. Gossan ${ }^{1}$, M. Gosselin ${ }^{28}$, R. Gouaty ${ }^{33}$, A. Grado ${ }^{5,124}$, C. Graef ${ }^{44}$, M. Granata ${ }^{22}$, A. Grant ${ }^{44}$, S. Gras ${ }^{12}$, P. Grassia ${ }^{1}$, C. Gray ${ }^{45}$, R. Gray ${ }^{44}$, G. Greco ${ }^{72,73}$, A. C. Green ${ }^{1,48}$, R. Green $^{68}$, E. M. Gretarsson ${ }^{34}$, P. Groot ${ }^{64}$, H. Grote ${ }^{68}$, S. Grunewald ${ }^{36}$, P. Gruning ${ }^{25}$, G. M. Guidi ${ }^{72,73}$, H. K. Gulati ${ }^{107}$, Y. Guo ${ }^{37}$, A. Gupta ${ }^{86}$,

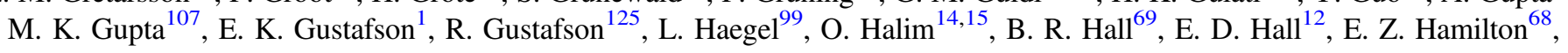


G. Hammond ${ }^{44}$, M. Haney ${ }^{66}$, M. M. Hanke ${ }^{8,9}$, J. Hanks ${ }^{45}$, C. Hanna ${ }^{86}$, O. A. Hannuksela ${ }^{90}$, J. Hanson ${ }^{7}$, T. Hardwick ${ }^{2}$, K. Haris ${ }^{16}$, J. Harms ${ }^{14,15}$, G. M. Harry ${ }^{126}$, I. W. Harry ${ }^{36}$, C.-J. Haster ${ }^{117}$, K. Haughian ${ }^{44}$, F. J. Hayes ${ }^{44}$, J. Healy ${ }^{58}$, A. Heidmann ${ }^{71}$, M. C. Heintze ${ }^{7}$, H. Heitmann ${ }^{65}$, P. Hello ${ }^{25}$, G. Hemming ${ }^{28}$, M. Hendry ${ }^{44}$ I. S. Heng ${ }^{44}$, J. Hennig ${ }^{8,9}$, A. W. Heptonstall ${ }^{1}$, F. J. Hernandez ${ }^{6}$, M. Heurs ${ }^{8,9}$, S. Hild ${ }^{44}$, T. Hinderer ${ }^{37,127,128}$, D. Hoak ${ }^{28}$, S. Hochheim ${ }^{8,9}$, D. Hofman ${ }^{22}$, A. M. Holgado ${ }^{17}$, N. A. Holland ${ }^{21}$, K. Holt ${ }^{7}$, D. E. Holz ${ }^{89}$, P. Hopkins ${ }^{68}$, C. Horst ${ }^{23}$, J. Hough ${ }^{44}$, E. J. Howell ${ }^{63}$, C. G. Hoy ${ }^{68}$, A. Hreibi ${ }^{65}$, E. A. Huerta ${ }^{17}$, D. Huet $^{25}$, B. Hughey ${ }^{34}$, M. Hulko ${ }^{1}$, S. Husa ${ }^{99}$, S. H. Huttner ${ }^{44}$, T. Huynh-Dinh ${ }^{7}$, B. Idzkowski ${ }^{74}$, A. Iess ${ }^{30,31}$, C. Ingram ${ }^{55}$, R. Inta ${ }^{83}$, G. Intini ${ }^{32,112}$, B. Irwin ${ }^{114}$, H. N. Isa ${ }^{44}$, J.-M. Isac ${ }^{71}$, M. Isi ${ }^{1}$, B. R. Iyer ${ }^{16}$, K. Izumi ${ }^{45}$, T. Jacqmin ${ }^{71}$, S. J. Jadhav ${ }^{129}$, K. Jani ${ }^{77}$, N. N. Janthalur ${ }^{129}$, P. Jaranowski ${ }^{130}$, A. C. Jenkins ${ }^{131}$, J. Jiang ${ }^{48}$, D. S. Johnson ${ }^{17}$, A. W. Jones ${ }^{11}$, D. I. Jones ${ }^{132}$, R. Jones ${ }^{44}$, R. J. G. Jonker ${ }^{37}$, L. Ju ${ }^{63}$, J. Junker ${ }^{8,9}$, C. V. Kalaghatgi ${ }^{68}$, V. Kalogera ${ }^{59}$, B. Kamai ${ }^{1}$, S. Kandhasamy ${ }^{84}$, G. Kang ${ }^{38}$, J. B. Kanner ${ }^{1}$, S. J. Kapadia ${ }^{23}$, S. Karki ${ }^{70}$, K. S. Karvinen ${ }^{8,9}$, R. Kashyap ${ }^{16}$, M. Kasprzack ${ }^{1}$, S. Katsanevas ${ }^{28}$, E. Katsavounidis ${ }^{12}$, W. Katzman ${ }^{7}$, S. Kaufer ${ }^{9}$, K. Kawabe ${ }^{45}$, N. V. Keerthana ${ }^{3}$, F. Kéfélian ${ }^{65}$, D. Keitel ${ }^{44}$, R. Kennedy ${ }^{108}$, J. S. Key ${ }^{133}$, F. Y. Khalilii ${ }^{62}$, H. Khan ${ }^{26}$, I. Khan ${ }^{14,31}$, S. Khan ${ }^{8,9}$, Z. Khan ${ }^{107}$, E. A. Khazanov ${ }^{134}$, M. Khursheed ${ }^{61}$, N. Kijbunchoo ${ }^{21}$, Chunglee $\mathrm{Kim}^{135}$, J. C. $\mathrm{Kim}^{136}$, K. Kim ${ }^{90}$, W. Kim${ }^{55}$, W. S. Kim ${ }^{137}$, Y.-M. Kim ${ }^{138}$, C. Kimball ${ }^{59}$, E. J. King ${ }^{55}$, P. J. King ${ }^{45}$, M. Kinley-Hanlon ${ }^{126}$, R. Kirchhoff ${ }^{8,9}$, J. S. Kissel ${ }^{45}$, L. Kleybolte ${ }^{139}$, J. H. Klika ${ }^{23}$, S. Klimenko ${ }^{48}$, T. D. Knowles ${ }^{39}$, P. Koch ${ }^{8,9}$, S. M. Koehlenbeck ${ }^{8,9}$, G. Koekoek ${ }^{37,140}$, S. Koley ${ }^{37}$, V. Kondrashov' ${ }^{1}$, A. Kontos ${ }^{12}$, N. Koper ${ }^{8,9}$, M. Korobko ${ }^{139}$, W. Z. Korth ${ }^{1}$,

I. Kowalska ${ }^{74}$, D. B. Kozak ${ }^{1}$, V. Kringel ${ }^{8,9}$, N. Krishnendu ${ }^{29}$, A. Królak ${ }^{141,142}$, G. Kuehn ${ }^{8,9}$, A. Kumar ${ }^{129}$, P. Kumar ${ }^{143}$,

R. Kumar ${ }^{107}$, S. Kumar ${ }^{16}$, L. Kuo ${ }^{87}$, A. Kutynia ${ }^{141}$, S. Kwang ${ }^{23}$, B. D. Lackey ${ }^{36}$, K. H. Lai ${ }^{90}$, T. L. Lam ${ }^{90}$, M. Landry ${ }^{4{ }^{4}}$,

B. B. Lane ${ }^{12}$, R. N. Lang ${ }^{144}$, J. Lange ${ }^{58}$, B. Lantz ${ }^{49}$, R. K. Lanza ${ }^{12}$, A. Lartaux-Vollard ${ }^{25}$, P. D. Lasky ${ }^{6}$, M. Laxen ${ }^{7}$, A. Lazzarini ${ }^{1}$, C. Lazzaro ${ }^{52}$, P. Leaci ${ }^{32,112}$, S. Leavey ${ }^{8,9}$, Y. K. Lecoeuche ${ }^{45}$, C. H. Lee ${ }^{92}$, H. K. Lee ${ }^{145}$, H. M. Lee ${ }^{146}$, H. W. Lee ${ }^{136}$, J. Lee ${ }^{91}$,

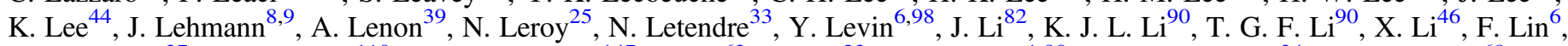
F. Linde ${ }^{37}$, S. D. Linker ${ }^{110}$, T. B. Littenberg ${ }^{147}$, J. Liu ${ }^{63}$, X. Liu ${ }^{23}$, R. K. L. Lo ${ }^{1,90}$, N. A. Lockerbie ${ }^{24}$, L. T. London ${ }^{68}$, A. Longo ${ }^{148,149}$, M. Lorenzini ${ }^{14,15}$, V. Loriette ${ }^{150}$, M. Lormand ${ }^{7}$, G. Losurdo ${ }^{19}$, J. D. Lough ${ }^{8,9}$, C. O. Lousto ${ }^{58}$, G. Lovelace ${ }^{26}$, M. E. Lower ${ }^{151}$, H. Lück ${ }^{8,9}$, D. Lumaca ${ }^{30,31}$, A. P. Lundgren ${ }^{152}$, R. Lynch ${ }^{12}$, Y. Ma ${ }^{46}$, R. Macas ${ }^{68}$, S. Macfoy ${ }^{24}$, M. MacInnis ${ }^{12}$, D. M. Macleod ${ }^{68}$, A. Macquet ${ }^{65}$, F. Magaña-Sandoval ${ }^{42}$, L. Magaña Zertuche ${ }^{84}$, R. M. Magee ${ }^{86}$, E. Majorana ${ }^{32}$, I. Maksimovic ${ }^{150}$, A. Malik ${ }^{61}$, N. Man $^{65}$, V. Mandic ${ }^{43}$, V. Mangano ${ }^{44}$, G. L. Mansell ${ }^{12,45}$, M. Manske ${ }^{21,23}$, M. Mantovani ${ }^{28}$, F. Marchesoni ${ }^{41,50}$, F. Marion ${ }^{33}$, S. Márka ${ }^{98}$, Z. Márka ${ }^{98}$, C. Markakis ${ }^{10,17}$, A. S. Markosyan ${ }^{49}$, A. Markowitz ${ }^{1}$, E. Maros ${ }^{1}$, A. Marquina ${ }^{102}$, S. Marsat $^{36}$, F. Martelli ${ }^{72,73}$, I. W. Martin ${ }^{44}$, R. M. Martin ${ }^{35}$, D. V. Martynov ${ }^{11}$, K. Mason ${ }^{12}$, E. Massera ${ }^{108}$, A. Masserot ${ }^{33}$, T. J. Massinger ${ }^{1}$, M. Masso-Reid ${ }^{44}$, S. Mastrogiovanni ${ }^{32,112}$, A. Matas ${ }^{36,43}$, F. Matichard ${ }^{1,12}$, L. Matone ${ }^{98}$, N. Mavalvala ${ }^{12}$, N. Mazumder ${ }^{69}$, J. J. McCann ${ }^{63}$, R. McCarthy ${ }^{45}$, D. E. McClelland ${ }^{21}$, S. McCormick ${ }^{7}$, L. McCuller ${ }^{12}$, S. C. McGuire ${ }^{153}$, J. McIver ${ }^{1}$,

D. J. McManus ${ }^{21}$, T. McRae ${ }^{21}$, S. T. McWilliams ${ }^{39}$, D. Meacher ${ }^{86}$, G. D. Meadors ${ }^{6}$, M. Mehmet ${ }^{8,9}$, A. K. Mehta ${ }^{16}$, J. Meidam $^{37}$, A. Melatos ${ }^{97}$, G. Mendell ${ }^{45}$, R. A. Mercer ${ }^{23}$, L. Mereni ${ }^{22}$, E. L. Merilh ${ }^{45}$, M. Merzougui ${ }^{65}$, S. Meshkov ${ }^{1}$, C. Messenger ${ }^{44}$, C. Messick $^{86}$, R. Metzdorff ${ }^{71}$, P. M. Meyers ${ }^{97}$, H. Miao ${ }^{11}$, C. Michel ${ }^{22}$, H. Middleton ${ }^{97}$, E. E. Mikhailov ${ }^{154}$, L. Milano ${ }^{5,79}$, A. L. Miller ${ }^{48}$, A. Miller ${ }^{32,112}$, M. Millhouse ${ }^{53}$, J. C. Mills ${ }^{68}$, M. C. Milovich-Goff ${ }^{110}$, O. Minazzoli ${ }^{65,155}$, Y. Minenkov ${ }^{31}$, A. Mishkin $^{48}$, C. Mishra ${ }^{156}$, T. Mistry ${ }^{108}$, S. Mitra ${ }^{3}$, V. P. Mitrofanov ${ }^{62}$, G. Mitselmakher ${ }^{48}$, R. Mittleman ${ }^{12}$, G. Mo ${ }^{93}$, D. Moffa $^{114}$, K. Mogushi ${ }^{84}$, S. R. P. Mohapatra ${ }^{12}$, M. Montani ${ }^{72,73}$, C. J. Moore ${ }^{10}$, D. Moraru ${ }^{45}$, G. Moreno ${ }^{45}$, S. Morisaki ${ }^{81}$, B. Mours ${ }^{33}$,

C. M. Mow-Lowry ${ }^{11}$, Arunava Mukherjee ${ }^{8,9}$, D. Mukherjee ${ }^{23}$, S. Mukherjee ${ }^{104}$, N. Mukund ${ }^{3}$, A. Mullavey ${ }^{7}$, J. Munch ${ }^{55}$,

E. A. Muñiz ${ }^{42}$, M. Muratore ${ }^{34}$, P. G. Murray ${ }^{44}$, A. Nagar ${ }^{85,157,158}$, I. Nardecchia ${ }^{30,31}$, L. Naticchioni ${ }^{32,112}$, R. K. Nayak ${ }^{159}$, J. Neilson ${ }^{110}$, G. Nelemans ${ }^{37,64}$, T. J. N. Nelson ${ }^{7}$, M. Nery, ${ }^{8,9}$ A. Neunzert ${ }^{125}$, K. Y. Ng $^{12},{\mathrm{~S} . ~ \mathrm{Ng}^{55}}^{5}$ P. Nguyen ${ }^{70}$, D. Nichols ${ }^{37,127}$, S. Nissanke ${ }^{37,127}$, F. Nocera ${ }^{28}$, C. North ${ }^{68}$, L. K. Nuttall ${ }^{152}$, M. Obergaulinger ${ }^{20}$, J. Oberling ${ }^{45}$, B. D. O'Brien ${ }^{48}$, G. D. O'Dea $^{110}$, G. H. Ogin ${ }^{160}$, J. J. Oh ${ }^{137}$, S. H. Oh ${ }^{137}$, F. Ohme ${ }^{8,9}$, H. Ohta ${ }^{81}$, M. A. Okada ${ }^{13}$, M. Oliver ${ }^{99}$, P. Oppermann ${ }^{8,9}$, Richard J. Oram ${ }^{7}$, B. O'Reilly ${ }^{7}$, R. G. Ormiston ${ }^{43}$, L. F. Ortega ${ }^{48}$, R. O'Shaughnessy ${ }^{58}$, S. Ossokine ${ }^{36}$, D. J. Ottaway ${ }^{55}$, H. Overmier ${ }^{7}$, B. J. Owen ${ }^{83}$, A. E. Pace ${ }^{86}$, G. Pagano ${ }^{18,19}$, M. A. Page ${ }^{63}$, A. Pai ${ }^{121}$, S. A. Pai ${ }^{61}$, J. R. Palamos ${ }^{70}$, O. Palashov ${ }^{134}$, C. Palomba ${ }^{32}$, A. Pal-Singh ${ }^{139}$, Huang-Wei Pan ${ }^{87}$, B. Pang ${ }^{46}$, P. T. H. Pang ${ }^{90}$, C. Pankow ${ }^{59}$, F. Pannarale ${ }^{32,112}$, B. C. Pant ${ }^{61}$, F. Paoletti ${ }^{19}$, A. Paoli ${ }^{28}$, A. Parida ${ }^{3}$, W. Parker ${ }^{7,153}$, D. Pascucci ${ }^{44}$, A. Pasqualetti ${ }^{28}$, R. Passaquieti ${ }^{18,19}$, D. Passuello ${ }^{19}$, M. Patil ${ }^{142}$, B. Patricelli ${ }^{18,19}$, B. L. Pearlstone ${ }^{44}$, C. Pedersen ${ }^{68}$, M. Pedraza ${ }^{1}$, R. Pedurand ${ }^{22,161}$, A. Pele ${ }^{7}$, S. Penn ${ }^{162}$, C. J. Perez ${ }^{45}$, A. Perreca ${ }^{95,111}$, H. P. Pfeiffer ${ }^{36,117}$, M. Phelps ${ }^{8,9}$, K. S. Phukon ${ }^{3}$, O. J. Piccinni ${ }^{32,112}$, M. Pichot ${ }^{65}$, F. Piergiovanni ${ }^{72,73}$, G. Pillant ${ }^{28}$, L. Pinard ${ }^{22}$, M. Pirello ${ }^{45}$, M. Pitkin ${ }^{44}$, R. Poggiani ${ }^{18,19}$, D. Y. T. Pong ${ }^{90}$, S. Ponrathnam ${ }^{3}$, P. Popolizio ${ }^{28}$, E. K. Porter ${ }^{27}$, J. Powell ${ }^{151}$, A. K. Prajapati ${ }^{107}$, J. Prasad ${ }^{3}$, K. Prasai ${ }^{49}$, R. Prasanna ${ }^{29}$, G. Pratten ${ }^{99}$, T. Prestegard ${ }^{23}$, S. Privitera ${ }^{36}$, G. A. Prodii ${ }^{95111}$, L. G. Prokhorov ${ }^{62}$, O. Puncken ${ }^{8,9}$, M. Punturo ${ }^{41}$, P. Puppo ${ }^{32}$, M. Pürrer ${ }^{36}$, H. Qi $^{23}$, V. Quetschke ${ }^{104}$, P. J. Quinonez ${ }^{34}$, E. A. Quintero ${ }^{1}$, R. Quitzow-James ${ }^{70}$,

F. J. Raab ${ }^{45}$, H. Radkins ${ }^{45}$, N. Radulescu ${ }^{65}$, P. Raffai ${ }^{106}$, S. Raja $^{61}$, C. Rajan ${ }^{61}$, B. Rajbhandari ${ }^{83}$, M. Rakhmanov ${ }^{104}$,

K. E. Ramirez ${ }^{104}$, A. Ramos-Buades ${ }^{99}$, Javed Rana ${ }^{3}$, K. Rao ${ }^{59}$, P. Rapagnani ${ }^{32,112}$, V. Raymond ${ }^{68}$, M. Razzano ${ }^{18,19}$, J. Read $^{26}$, T. Regimbau ${ }^{33}$, L. Rei ${ }^{60}$, S. Reid ${ }^{24}$, D. H. Reitze ${ }^{1,48}$, W. $\operatorname{Ren}^{17}$, F. Ricci ${ }^{32,112}$, C. J. Richardson ${ }^{34}$, J. W. Richardson ${ }^{1}$, P. M. Ricker ${ }^{17}$, K. Riles ${ }^{125}$, M. Rizzo ${ }^{59}$, N. A. Robertson ${ }^{1,44}$, R. Robie ${ }^{44}$, F. Robinet ${ }^{25}$, A. Rocchi ${ }^{31}$, L. Rolland ${ }^{33}$, J. G. Rollins ${ }^{1}$, V. J. Roma ${ }^{70}$, M. Romanelli ${ }^{67}$, R. Romano ${ }^{4,5}$, C. L. Romel ${ }^{45}$, J. H. Romie ${ }^{7}$, K. Rose ${ }^{114}$, D. Rosińska ${ }^{54,163}$, S. G. Rosofsky ${ }^{17}$, M. P. Ross ${ }^{164}{ }^{\text {, }}$ S. Rowan ${ }^{44}$, A. Rüdiger ${ }^{8,9,178}$, P. Ruggi ${ }^{28}$, G. Rutins ${ }^{165}$, K. Ryan ${ }^{45}$, S. Sachdev ${ }^{1}$, T. Sadecki ${ }^{45}$, M. Sakellariadou ${ }^{131}$, L. Salconi ${ }^{28}$, M. Saleem ${ }^{29}$, A. Samajdar ${ }^{37}$, L. Sammut ${ }^{6}$, E. J. Sanchez ${ }^{1}$, L. E. Sanchez ${ }^{1}$, N. Sanchis-Gual ${ }^{20}$, V. Sandberg ${ }^{45}$, J. R. Sanders ${ }^{42}$, K. A. Santiago ${ }^{35}$, N. Sarin $^{6}$, B. Sassolas ${ }^{22}$, P. R. Saulson ${ }^{42}$, O. Sauter ${ }^{125}$, R. L. Savage ${ }^{45}$, P. Schale ${ }^{70}$, M. Scheel ${ }^{46}$ J. Scheuer $^{59}$, 
P. Schmidt ${ }^{64}$, R. Schnabel ${ }^{139}$, R. M. S. Schofield ${ }^{70}$, A. Schönbeck ${ }^{139}$, E. Schreiber ${ }^{8,9}$, B. W. Schulte ${ }^{8,9}$, B. F. Schutz ${ }^{68}$, S. G. Schwalbe ${ }^{34}$, J. Scott ${ }^{44}$, S. M. Scott ${ }^{21}$, E. Seidel ${ }^{17}$, D. Sellers ${ }^{7}$, A. S. Sengupta ${ }^{166}$, N. Sennett ${ }^{36}$, D. Sentenac ${ }^{28}$, V. Sequino ${ }^{14,30,31}$, A. Sergeev ${ }^{134}$, Y. Setyawati ${ }^{8,9}$, D. A. Shaddock ${ }^{21}$, T. Shaffer ${ }^{45}$, M. S. Shahriar ${ }^{59}$, M. B. Shaner ${ }^{110}$, L. Shao ${ }^{36}$, P. Sharma ${ }^{61}$, P. Shawhan ${ }^{76}$, H. Shen ${ }^{17}$, R. Shink ${ }^{167}$, D. H. Shoemaker ${ }^{12}$, D. M. Shoemaker ${ }^{77}$, S. ShyamSundar ${ }^{61}$, K. Siellez ${ }^{77}$, M. Sieniawska ${ }^{54}$, D. $\operatorname{Sigg}^{45}$, A. D. Silva ${ }^{13}$, L. P. Singer $^{80}$, N. $\operatorname{Singh}^{74}$, A. Singhal ${ }^{14,32}$, A. M. Sintes ${ }^{99}$, S. Sitmukhambetov ${ }^{104}$, V. Skliris ${ }^{68}$, B. J. J. Slagmolen ${ }^{21}$, T. J. Slaven-Blair ${ }^{63}$, J. R. Smith ${ }^{26}$, R. J. E. Smith ${ }^{6}$, S. Somala ${ }^{168}$, E. J. Son ${ }^{137}$, B. Sorazu ${ }^{44}$, F. Sorrentino ${ }^{60}$, T. Souradeep ${ }^{3}$, E. Sowell ${ }^{83}$, A. P. Spencer ${ }^{44}$, A. K. Srivastava ${ }^{107}$, V. Srivastava ${ }^{42}$, K. Staats ${ }^{59}$, C. Stachie ${ }^{65}$, M. Standke ${ }^{8,9}$, D. A. Steer ${ }^{27}$, M. Steinke ${ }^{8,9}$, J. Steinlechner ${ }^{44,139}$, S. Steinlechner ${ }^{139}$, D. Steinmeyer ${ }^{8,9}$, S. P. Stevenson ${ }^{151}$, D. Stocks ${ }^{49}$, R. Stone ${ }^{104}$, D. J. Stops ${ }^{11}$, K. A. Strain ${ }^{44}$, G. Stratta ${ }^{72,73}$, S. E. Strigin ${ }^{62}$, A. Strunk ${ }^{45}$, R. Sturani ${ }^{169}$, A. L. Stuver ${ }^{170}$, V. Sudhir ${ }^{12}$, T. Z. Summerscales ${ }^{171}$, L. Sun ${ }^{1}$, S. Sunil ${ }^{107}$, J. Suresh ${ }^{3}$, P. J. Sutton ${ }^{68}$, B. L. Swinkels ${ }^{37}$, M. J. Szczepańczyk ${ }^{34}$, M. Tacca $^{37}$, S. C. Tait ${ }^{44}$, C. Talbot ${ }^{6}$, D. Talukder ${ }^{70}$, D. B. Tanner ${ }^{48}$, M. Tápai ${ }^{122}$, A. Taracchini ${ }^{36}$, J. D. Tasson ${ }^{93}$, R. Taylor ${ }^{1}$, F. Thies ${ }^{8,9}$, M. Thomas ${ }^{7}$, P. Thomas ${ }^{45}$, S. R. Thondapu ${ }^{61}$, K. A. Thorne ${ }^{7}$, E. Thrane ${ }^{6}$, Shubhanshu Tiwari ${ }^{95,111}$, Srishti Tiwari ${ }^{123}$, V. Tiwari ${ }^{68}$, K. Toland ${ }^{44}$, M. Tonelli ${ }^{18,19}$, Z. Tornasi ${ }^{44}$, A. Torres-Forné ${ }^{172}$, C. I. Torrie ${ }^{1}$, D. Töyrä ${ }^{11}$, F. Travasso ${ }^{28,41}$, G. Traylor $^{7}$,

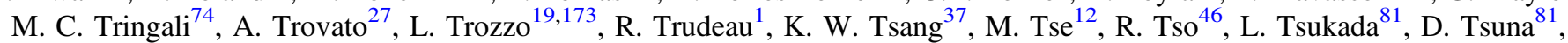
D. Tuyenbayev ${ }^{104}$, K. Ueno ${ }^{81}$, D. Ugolini ${ }^{174}$, C. S. Unnikrishnan ${ }^{123}$, A. L. Urban ${ }^{2}$, S. A. Usman ${ }^{68}$, H. Vahlbruch ${ }^{9}$, G. Vajente ${ }^{1}$, G. Valdes ${ }^{2}$, N. van Bakel ${ }^{37}$, M. van Beuzekom ${ }^{37}$, J. F. J. van den Brand ${ }^{37,75}$, C. Van Den Broeck ${ }^{37,175}$, D. C. Vander-Hyde ${ }^{42}$, L. van der Schaaf ${ }^{37}$, J. V. van Heijningen ${ }^{63}$, A. A. van Veggel ${ }^{44}$, M. Vardaro ${ }^{51,52}$, V. Varma ${ }^{46}$, S. Vass ${ }^{1}$, M. Vasúth ${ }^{47}$, A. Vecchio ${ }^{11}$, G. Vedovato ${ }^{52}$, J. Veitch ${ }^{44}$, P. J. Veitch ${ }^{55}$, K. Venkateswara ${ }^{164}$, G. Venugopalan ${ }^{1}$, D. Verkindt ${ }^{33}$, F. Vetrano ${ }^{72,73}$, A. Viceré ${ }^{72,73}$, A. D. Viets ${ }^{23}$, D. J. Vine ${ }^{165}$, J.-Y. Vinet ${ }^{65}$, S. Vitale ${ }^{12}$, T. Vo ${ }^{42}$, H. Vocca $^{40,41}$, C. Vorvick ${ }^{45}$, S. P. Vyatchanin ${ }^{62}$, A. R. Wade ${ }^{1}$, L. E. Wade ${ }^{114}$, M. Wade ${ }^{114}$, R. Walet ${ }^{37}$, M. Walker ${ }^{26}$, L. Wallace ${ }^{1}$, S. Walsh ${ }^{23}$, G. Wang ${ }^{14,19}$, H. Wang ${ }^{11}$, J. Z. Wang ${ }^{125}$, W. H. Wang ${ }^{104}$, Y. F. Wang ${ }^{90}$, R. L. Ward ${ }^{21}$, Z. A. Warden ${ }^{34}$, J. Warner ${ }^{45}$, M. Was ${ }^{33}$, J. Watchi ${ }^{100}$, B. Weaver ${ }^{45}$, L.-W. Wei ${ }^{8,9}$, M. Weinert ${ }^{8,9}$, A. J. Weinstein ${ }^{1}$, R. Weiss ${ }^{12}$, F. Wellmann ${ }^{8,9}$, L. Wen ${ }^{63}$, E. K. Wessel ${ }^{17}$, P. Weßels ${ }^{8,9}$, J. W. Westhouse ${ }^{34}$, K. Wette ${ }^{21}$, J. T. Whelan ${ }^{58}$, B. F. Whiting ${ }^{48}$, C. Whittle ${ }^{12}$, D. M. Wilken ${ }^{8,9}$, D. Williams ${ }^{44}$, A. R. Williamson ${ }^{37,127}$, J. L. Willis ${ }^{1}$, B. Willke ${ }^{8,9}$, M. H. Wimmer ${ }^{8,9}$, W. Winkler, ${ }^{8,9}$, C. C. Wipf ${ }^{1}$, H. Wittel ${ }^{8,9}$, G. Woan ${ }^{44}$, J. Woehler ${ }^{8,9}$, J. K. Wofford ${ }^{58}$, J. Worden ${ }^{45}$, J. L. Wright ${ }^{44}$, D. S. Wu ${ }^{8,9}$, D. M. Wysocki ${ }^{58}$, L. Xiao ${ }^{1}$, H. Yamamoto ${ }^{1}$, C. C. Yancey ${ }^{76}$, L. Yang ${ }^{113}$, M. J. Yap ${ }^{21}$, M. Yazback ${ }^{48}$, D. W. Yeeles ${ }^{68}$, Hang Yu ${ }^{12}$, Haocun $\mathrm{Yu}^{12}$, S. H. R. Yuen ${ }^{90}$, M. Yvert ${ }^{33}$, A. K. Zadrożny ${ }^{104,141}$, M. Zanolin ${ }^{34}$, T. Zelenova ${ }^{28}$, J.-P. Zendri ${ }^{52}$, M. Zevin ${ }^{59}$, J. Zhang ${ }^{63}$, L. Zhang ${ }^{1}$, T. Zhang ${ }^{44}$, C. Zhao ${ }^{63}$, M. Zhou ${ }^{59}$, Z. Zhou ${ }^{59}$, X. J. Zhu ${ }^{6}$, M. E. Zucker ${ }^{1,12}$, and J. Zweizig ${ }^{1}$

(The LIGO Scientific Collaboration and the Virgo Collaboration)

${ }^{1}$ LIGO, California Institute of Technology, Pasadena, CA 91125, USA

${ }^{2}$ Louisiana State University, Baton Rouge, LA 70803, USA

${ }^{3}$ Inter-University Centre for Astronomy and Astrophysics, Pune 411007, India

${ }^{4}$ Università di Salerno, Fisciano, I-84084 Salerno, Italy

5 INFN, Sezione di Napoli, Complesso Universitario di Monte S.Angelo, I-80126 Napoli, Italy

${ }^{6} \mathrm{OzGrav}$, School of Physics \& Astronomy, Monash University, Clayton, VIC 3800, Australia ${ }^{7}$ LIGO Livingston Observatory, Livingston, LA 70754, USA

${ }^{8}$ Max Planck Institute for Gravitational Physics (Albert Einstein Institute), D-30167 Hannover, Germany

${ }^{9}$ Leibniz Universität Hannover, D-30167 Hannover, Germany

${ }^{10}$ University of Cambridge, Cambridge CB2 1TN, UK

${ }^{11}$ University of Birmingham, Birmingham B15 2TT, UK

${ }^{12}$ LIGO, Massachusetts Institute of Technology, Cambridge, MA 02139, USA

${ }^{13}$ Instituto Nacional de Pesquisas Espaciais, 12227-010 São José dos Campos, São Paulo, Brazil ${ }^{14}$ Gran Sasso Science Institute (GSSI), I-67100 L'Aquila, Italy

${ }^{15}$ INFN, Laboratori Nazionali del Gran Sasso, I-67100 Assergi, Italy

${ }^{16}$ International Centre for Theoretical Sciences, Tata Institute of Fundamental Research, Bengaluru 560089, India

${ }^{17}$ NCSA, University of Illinois at Urbana-Champaign, Urbana, IL 61801, USA

${ }^{18}$ Università di Pisa, I-56127 Pisa, Italy

${ }^{19}$ INFN, Sezione di Pisa, I-56127 Pisa, Italy

${ }^{20}$ Departamento de Astronomía y Astrofísica, Universitat de València, E-46100 Burjassot, València, Spain

${ }^{21}$ OzGrav, Australian National University, Canberra, ACT 0200, Australia

${ }^{22}$ Laboratoire des Matériaux Avancés (LMA), CNRS/IN2P3, F-69622 Villeurbanne, France

${ }^{23}$ University of Wisconsin-Milwaukee, Milwaukee, WI 53201, USA

${ }^{24}$ SUPA, University of Strathclyde, Glasgow G1 1XQ, UK
${ }^{25}$ LAL, Univ. Paris-Sud, CNRS/IN2P3, Université Paris-Saclay, F-91898 Orsay, France

${ }^{26}$ California State University Fullerton, Fullerton, CA 92831, USA

${ }^{27}$ APC, AstroParticule et Cosmologie, Université Paris Diderot, CNRS/IN2P3, CEA/Irfu, Observatoire de Paris, Sorbonne Paris Cité, F-75205 Paris Cedex 13, France

${ }^{28}$ European Gravitational Observatory (EGO), I-56021 Cascina, Pisa, Italy

${ }_{29}$ Chennai Mathematical Institute, Chennai 603103, India

${ }^{30}$ Università di Roma Tor Vergata, I-00133 Roma, Italy

${ }^{31}$ INFN, Sezione di Roma Tor Vergata, I-00133 Roma, Italy

32 INFN, Sezione di Roma, I-00185 Roma, Italy

${ }^{33}$ Laboratoire d'Annecy de Physique des Particules (LAPP), Univ. Grenoble Alpes, Université Savoie Mont Blanc, CNRS/IN2P3, F-74941 Annecy, France

${ }^{34}$ Embry-Riddle Aeronautical University, Prescott, AZ 86301, USA

${ }^{35}$ Montclair State University, Montclair, NJ 07043, USA

${ }^{36}$ Max Planck Institute for Gravitational Physics (Albert Einstein Institute), D-14476 Potsdam-Golm, Germany

${ }^{37}$ Nikhef, Science Park 105, 1098 XG Amsterdam, The Netherlands 


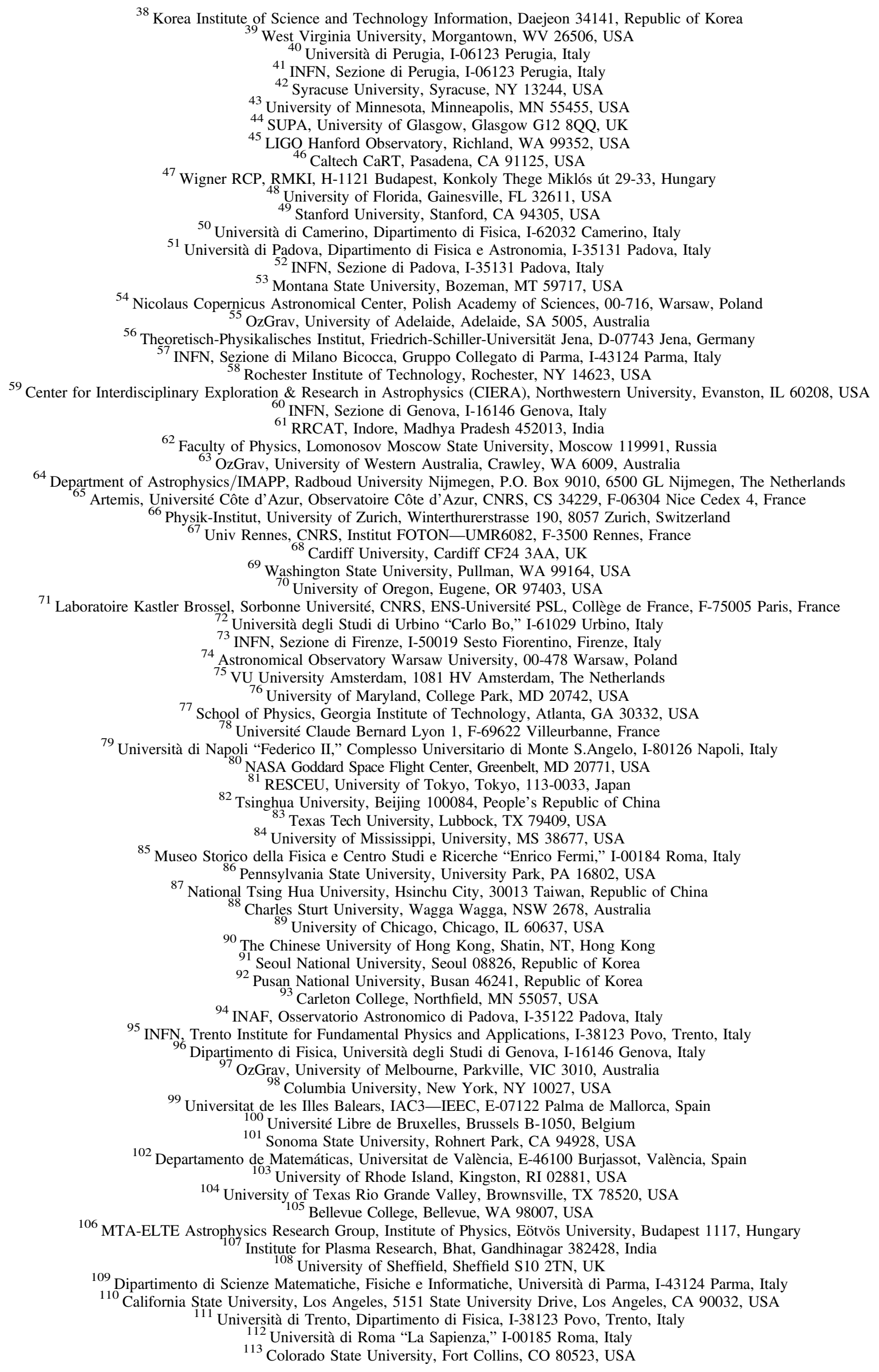




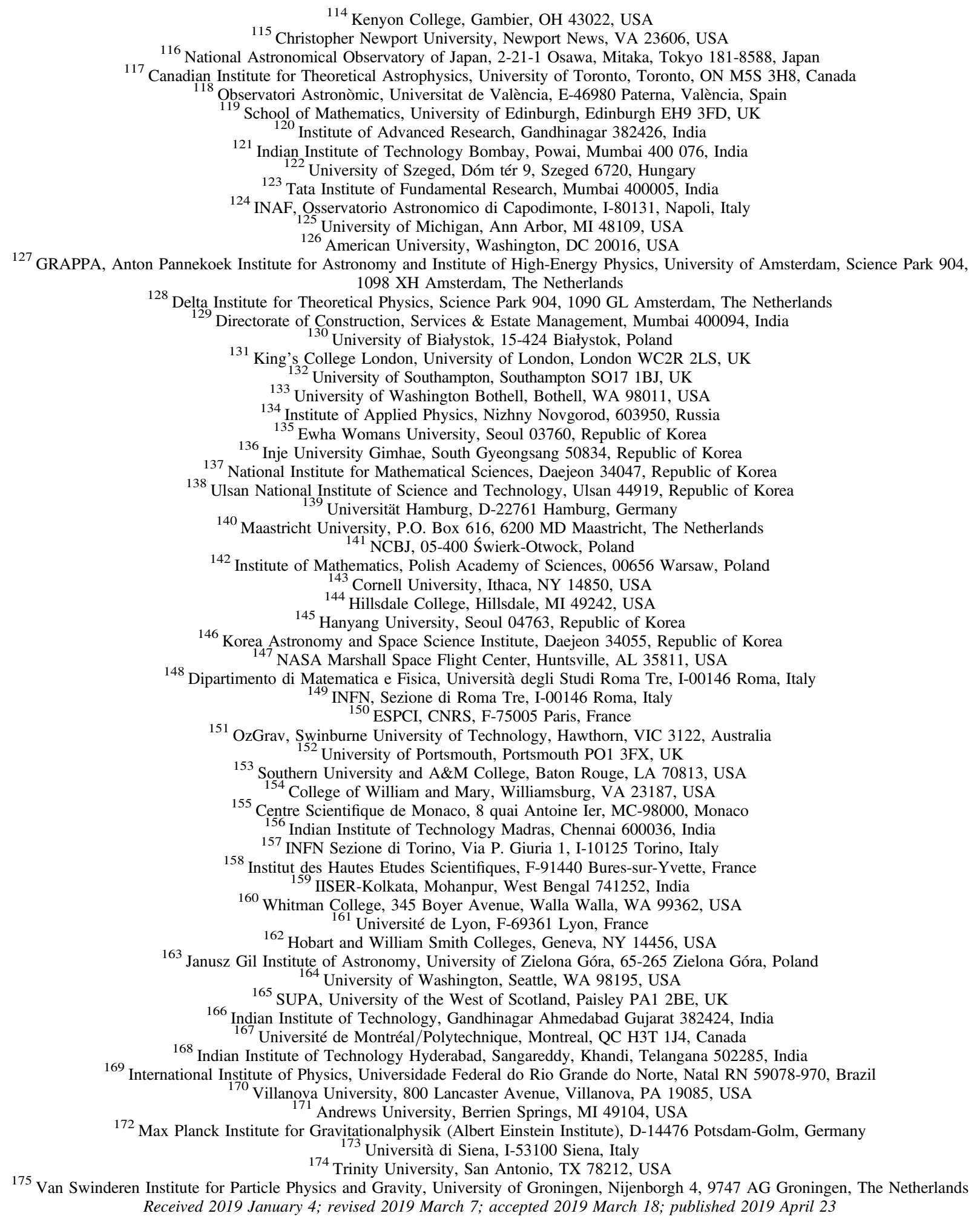

\footnotetext{
* Any correspondence should be addressed to 1sc-spokesperson@ligo.org and virgo-spokesperson@ego-gw.it..

176 Deceased, 2018 February.

177 Deceased, 2017 November.

178 Deceased, 2018 July.
} 


\begin{abstract}
We describe directed searches for continuous gravitational waves (GWs) from 16 well-localized candidate neutron stars, assuming none of the stars has a binary companion. The searches were directed toward 15 supernova remnants and Fomalhaut b, a directly imaged extrasolar planet candidate that has been suggested to be a nearby old neutron star. Each search covered a broad band of frequencies and first and second time derivatives. After coherently integrating spans of data from the first Advanced LIGO observing run of 3.5-53.7 days per search, applying data-based vetoes, and discounting known instrumental artifacts, we found no astrophysical signals. We set upper limits on intrinsic GW strain as strict as $1 \times 10^{-25}$, fiducial neutron star ellipticity as strict as $2 \times 10^{-9}$, and fiducial $r$-mode amplitude as strict as $3 \times 10^{-8}$.
\end{abstract}

Key words: gravitational waves - ISM: supernova remnants - stars: neutron

\section{Introduction}

With the detections of several binary black hole mergers (Abbott et al. 2016b, 2016c, 2017e, 2017f, 2017g) and one binary neutron star merger (Abbott et al. 2017h) also seen in electromagnetic waves (Abbott et al. 2017i), Advanced LIGO and Virgo have spectacularly inaugurated the field of gravitational wave (GW) astronomy. While the binary neutron star merger has had farreaching implications for our knowledge of neutron star matter (Abbott et al. 2018b; De et al. 2018b), a continuous GW signal could teach us even more-not just about bulk properties but about internal magnetic fields, the extent and strength of crystalline phases, and, potentially, other microphysics of extreme matter (Owen 2009; Glampedakis \& Gualtieri 2018).

Young isolated neutron stars are promising sources of continuous GWs. The spin-downs of young pulsars are rapid enough to include significant continuous GW emission, as shown by the latest GW search for known pulsars (Abbott et al. 2017c, 2017d). Theoretical arguments suggest that $r$-modes (oscillations dominated by the Coriolis force) might remain unstable and detectable in neutron stars up to a few thousand yr old (Owen 2010, and references therein). Most young supernova remnants (SNRs) do not contain known pulsars (Green 2014). On the other hand, many of these SNRs contain small pulsar wind nebulae (PWNe), central compact objects (CCOs), or other welllocalized nonpulsing candidate neutron stars. Also, some of these SNRs are young enough that a neutron star could not have been kicked far, and thus the star can be considered well localized even if it is not seen at all. A GW search directed at a single sky position can significantly improve on the sensitivities of all-sky surveys, even while needing to cover a wide band of possible GW frequencies and first and second time derivatives due to a lack of pulsations from the object (Wette et al. 2008). This makes nonpulsing isolated neutron stars attractive targets for continuous GW searches if they are well localized.

Directed GW searches for isolated neutron stars have been published targeting SNRs (Abadie et al. 2010, 2011; Aasi et al. 2015; Sun et al. 2016; Zhu et al. 2016; Abbott et al. 2017b) and promising locations, including the Galactic center (Abadie et al. 2011; Aasi et al. 2013; Abbott et al. 2017b) and the core of a nearby globular cluster, where multibody interactions might effectively rejuvenate some neutron stars' continuous GW emission (Abbott et al. 2017j). The only such search of data from advanced interferometers so far (Abbott et al. 2017b) employed methods from stochastic background searches, which, while quick to implement, are not as sensitive as continuous wave search methods.

Here we present the first directed continuous wave searches for isolated nonpulsing neutron stars in data from the first Advanced LIGO observing run (O1). We used an extension of the coherent data analysis pipeline used in Abadie et al. (2010) and
Aasi et al. (2015), to which this paper is a sequel. The improved noise curve (with respect to initial LIGO and Virgo) means that we can search more targets with sensitivity beating the indirect upper limit on GW emission due to energy conservation (Wette et al. 2008) based on the age of the neutron star (similar to the spin-down limit for known pulsars). We include not only more SNRs but also the directly imaged exoplanet candidate Fomalhaut b, which has been proposed to be an old nearby neutron star (Neuhäuser et al. 2015)—close enough that it is an attractive target in spite of being much older than the others. We do not include SN 1987A because it is so young that the possible spin-down parameter space is too large to cover with a coherent wideband search and reasonable computational cost.

\section{Searches}

\subsection{Methods}

These searches were based on the multi-interferometer $\mathcal{F}$-statistic (Jaranowski et al. 1998; Cutler \& Schutz 2005). The $\mathcal{F}$-statistic accounts for the modulation of the signal due to the daily rotation of the detectors by adding the outputs of sinusoidal matched filters in quadrature. For these searches, the frequency evolution of each filter in the reference frame of the solar system barycenter was given by

$$
f(t)=f+\dot{f}\left(t-t_{0}\right)+\frac{1}{2} \ddot{f}\left(t-t_{0}\right)^{2},
$$

where $t_{0}$ is the beginning of the observation, the frequency derivatives are evaluated at that time, and, in a slight abuse of notation, we use a simple $f$ for $f\left(t_{0}\right)$. Hence, these filters are designed to detect neutron stars without binary companions whose spin-down is not too fast (requiring third or higher frequency derivatives) or too irregular (having significant timing noise or glitches) during the observation. In stationary Gaussian noise, $2 \mathcal{F}$ is drawn from a $\chi^{2}$ distribution with four degrees of freedom, which for loud signals makes the amplitude signal-to-noise ratio roughly $\sqrt{\mathcal{F} / 2}$. If a signal is present, the $\chi^{2}$ is noncentral.

We used data from LIGO O1 but none from Virgo because that interferometer was down for upgrades during O1. At the frequencies to which LIGO was most sensitive (about $100-300 \mathrm{~Hz}$ ), the strain noise amplitude was about three to four times lower than in the sixth LIGO science run (S6; Abbott et al. 2016a). However, there were many more spectral lines due to instrumental artifacts than in S6, which complicated the analysis. We used the calibration described in Abbott et al. (2017d), which is an update of the first O1 calibration described in Abbott et al. (2017a). Hence, as in Abbott et al. (2017d), our upper limits on strain are uncertain by at least $14 \%$. Like many other continuous $\mathrm{GW}$ searches, ours used data in the form of short 
Table 1

Targeted Objects and Astronomical Parameters Used in Each Search

\begin{tabular}{|c|c|c|c|c|c|}
\hline $\begin{array}{l}\text { SNR } \\
\text { (G Name) }\end{array}$ & $\begin{array}{l}\text { Parameter } \\
\text { Space }\end{array}$ & Other Name & $\begin{array}{l}\text { R.A.+decl. } \\
\quad(\text { J2000) }\end{array}$ & $\begin{array}{c}D \\
(\mathrm{kpc})\end{array}$ & $\begin{array}{l}a \\
(\mathrm{kyr})\end{array}$ \\
\hline $1.9+0.3$ & $\ldots$ & $\ldots$ & $174846.9-271016$ & 8.5 & 0.1 \\
\hline $15.9+0.2$ & $\cdots$ & $\cdots$ & $181852.1-150214$ & 8.5 & 0.54 \\
\hline $18.9-1.1$ & $\ldots$ & $\ldots$ & $182913.1-125113$ & 2 & 4.4 \\
\hline $39.2-0.3$ & $\ldots$ & 3 C 396 & $190404.7+052712$ & 6.2 & 3 \\
\hline $65.7+1.2$ & $\ldots$ & DA 495 & $195217.0+292553$ & 1.5 & 20 \\
\hline $93.3+6.9$ & $\ldots$ & DA 530 & $205214.0+551722$ & 1.7 & 5 \\
\hline $111.7-2.1$ & $\cdots$ & Cas A & $232327.9+584842$ & 3.3 & 0.3 \\
\hline $189.1+3.0$ & Wide & IC 443 & $061705.3+222127$ & 1.5 & 3 \\
\hline $189.1+3.0$ & Deep & IC 443 & $061705.3+222127$ & 1.5 & 20 \\
\hline $266.2-1.2$ & Wide & Vela Jr. & $085201.4-461753$ & 0.2 & 0.69 \\
\hline $266.2-1.2$ & Deep & Vela Jr. & $085201.4-461753$ & 0.9 & 5.1 \\
\hline $291.0-0.1$ & $\cdots$ & MSH 11-62 & $111148.6-603926$ & 3.5 & 1.2 \\
\hline $330.2+1.0$ & $\ldots$ & $\cdots$ & $160103.1-513354$ & 5 & 1 \\
\hline $347.3-0.5$ & $\cdots$ & $\cdots$ & $171328.3-394953$ & 0.9 & 1.6 \\
\hline $350.1-0.3$ & $\ldots$ & $\cdots$ & $172054.5-372652$ & 4.5 & 0.6 \\
\hline $353.6-0.7$ & $\ldots$ & $\ldots$ & $173203.3-344518$ & 3.2 & 27 \\
\hline $354.4+0.0$ & Wide & $\cdots$ & $173127.5-333412$ & 5 & 0.1 \\
\hline $354.4+0.0$ & Deep & $\cdots$ & $173127.5-333412$ & 8 & 0.5 \\
\hline$\ldots$ & Wide & Fomalhaut b & $225739.1-293720$ & 0.011 & 316 \\
\hline$\cdots$ & Deep & Fomalhaut b & $225739.1-293720$ & 0.02 & 3000 \\
\hline
\end{tabular}

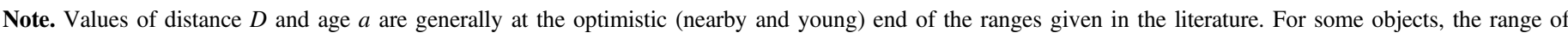

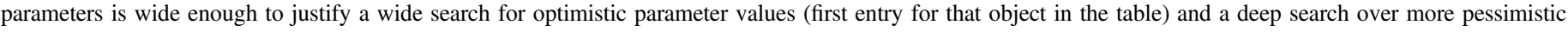
parameter values (second entry). See text for details and references.

Fourier transforms (SFTs) of duration $1800 \mathrm{~s}$, high-pass-filtered and Tukey-windowed to reduce artifacts, recording only frequencies up to $2 \mathrm{kHz}$.

While each search targeted a specific direction (R.A. and decl.), each had to cover a broad band of frequencies and first and second derivatives. That is, a bank of signal templates was required, constructed to cover the parameter space $(f, \dot{f}, \ddot{f})$ with sufficient density (Whitbeck 2006; Wette et al. 2008). We chose coverage such that the maximum loss of power signal-to-noise ratio due to mismatch between the signal and the nearest template (Owen 1996; Brady et al. 1998) was no worse than $20 \%$, a common choice in continuous GW analyses. Given the parameter choices described below, this resulted in $10^{12}-10^{13}$ templates for most searches, with the Cas A search getting more than $10^{14}$, since it was allocated 10 times the computing cycles of the other searches.

All searches ran on the Atlas computing cluster at the Max Planck Institute for Gravitational Physics (Albert Einstein Institute) in Hanover, Germany, using the same tag (S6SNRSearch) of the LALSuite software package (LIGO Scientific Collaboration 2018) as in Aasi et al. (2015), although the controlling scripts were upgraded. Most searches used roughly $10^{5}$ core hours (split into roughly $3 \times 10^{4}$ batch jobs), and Cas A used more than $10^{6}$ (split into roughly $3 \times 10^{5}$ jobs). The splitting into jobs was used in the vetoes and other post-processing described in Section 2.4. Post-processing for each search used at most of order $10 \%$ of the core hours dedicated to the search. Several terabytes of search results were written to disk.

\subsection{Target List}

Our choice of targets required that a search of the fixed computational cost had to be sensitive enough to detect the strongest continuous GW signal consistent with broad conservation of energy considerations. As introduced by Wette et al. (2008) for the CCO in SNR Cas A, the strongest possible signal based on the age $a$ and distance $D$ of the source,

$$
h_{0}^{\text {age }}=1.26 \times 10^{-24}\left(\frac{3.30 \mathrm{kpc}}{D}\right)\left(\frac{300 \mathrm{yr}}{a}\right)^{1 / 2},
$$

is analogous to the spin-down limit for known pulsars and indicates the strongest possible intrinsic strain produced by an object whose unknown spin-down is entirely due to GW emission and has been since birth. The fiducial parameters (for Cas A) show that this limit can be high enough to be interesting. The intrinsic strain $h_{0}$ (Jaranowski et al. 1998) characterizes the GW metric perturbation without reference to any particular orientation or polarization and hence is typically a factor of 2-3 greater than the strain response measured by the interferometers. The indirect limit $h_{0}^{\text {age }}$ is slightly different for $r$-mode emission (Owen 2010) than for the mass quadrupole source tacitly assumed above and in most of the literature, but we neglect this small difference. Due to uncertainties in the neutron star mass and equation of state, $h_{0}^{\text {age }}$ is uncertain by of order $50 \%$, which we also neglect.

To choose directions to search, we started from the Green catalog of SNRs (Green 2014). We picked X-ray point sources (CCOs or candidate CCOs), small PWNe, and, in some cases, relatively young SNRs, where any neutron star could not yet have moved far. We selected only targets with age and distance estimates so that we could evaluate $h_{0}^{\text {age }}$. In some cases, there is a wide range of estimates in the literature, leading to significant differences in $h_{0}^{\text {age }}$. In most cases, we used the most optimistic estimates, yielding the highest $h_{0}^{\text {age }}$ but also the most difficult search over the widest band of frequency and spin-down parameters. In addition to this wide search using the optimistic age and distance, we did a deep search using the most pessimistic age and distance in cases where the strain 
Table 2

Derived Parameters Used in Each Search

\begin{tabular}{|c|c|c|c|c|c|c|c|c|c|c|}
\hline $\begin{array}{l}\text { SNR } \\
\text { (G Name) }\end{array}$ & $\begin{array}{l}\text { Parameter } \\
\text { Space }\end{array}$ & $\begin{array}{l}f_{\min } \\
(\mathrm{Hz})\end{array}$ & $\begin{array}{l}f_{\max } \\
(\mathrm{Hz})\end{array}$ & $\begin{array}{c}T_{\text {span }} \\
\text { (s) }\end{array}$ & $\begin{array}{c}T_{\text {span }} \\
\text { (days) }\end{array}$ & $\begin{array}{l}\text { Start of Span } \\
\text { (UTC, 2015) }\end{array}$ & $\begin{array}{l}\text { H1 } \\
\text { SFTs }\end{array}$ & $\begin{array}{c}\text { L1 } \\
\text { SFTs }\end{array}$ & $\begin{array}{l}\text { Duty } \\
\text { Factor }\end{array}$ & $\begin{array}{l}h_{0}^{\text {age }} \\
\left(\times 10^{-25}\right)\end{array}$ \\
\hline $1.9+0.3$ & $\cdots$ & 38 & 1332 & 336,307 & 3.9 & Nov 30 03:53:08 & 156 & 141 & 0.79 & 8.4 \\
\hline $15.9+0.2$ & $\ldots$ & 72 & 538 & 887,744 & 10.3 & Nov 25 13:39:16 & 369 & 304 & 0.68 & 3.6 \\
\hline $39.2-0.3$ & $\ldots$ & 98 & 295 & $1,965,780$ & 22.8 & Nov 28 00:47:19 & 641 & 647 & 0.59 & 2.1 \\
\hline $65.7+1.2$ & $\ldots$ & 53 & 794 & $1,932,067$ & 22.4 & Dec 14 04:52:40 & 774 & 555 & 0.62 & 3.4 \\
\hline $93.3+6.9$ & $\ldots$ & 41 & 1215 & $1,051,764$ & 12.2 & Nov 25 12:39:16 & 385 & 354 & 0.63 & 5.9 \\
\hline $189.1+3.0$ & Deep & 50 & 805 & $1,933,867$ & 22.4 & Dec 14 04:52:40 & 775 & 555 & 0.62 & 3.4 \\
\hline $266.2-1.2$ & Wide & 19 & 1998 & 462,616 & 5.4 & Nov 28 02:17:19 & 191 & 213 & 0.79 & 136 \\
\hline $266.2-1.2$ & Deep & 32 & 1998 & 799,819 & 9.3 & Nov 26 12:43:17 & 329 & 294 & 0.70 & 11.2 \\
\hline $291.0-0.1$ & $\ldots$ & 42 & 987 & 788,409 & 9.1 & Nov 26 18:28:03 & 322 & 295 & 0.70 & 5.9 \\
\hline $330.2+1.0$ & $\ldots$ & 53 & 731 & 851,744 & 9.9 & Nov 25 23:39:16 & 349 & 302 & 0.69 & 4.5 \\
\hline $347.3-0.5$ & $\ldots$ & 27 & 1998 & 578,325 & 6.7 & Nov 28 05:17:19 & 237 & 253 & 0.76 & 19.9 \\
\hline Fomalhaut b & Wide & 19 & 1998 & $2,492,267$ & 28.8 & Sep 18 20:08:24 & 955 & 799 & 0.63 & 116 \\
\hline Fomalhaut b & Deep & 22 & 1998 & $4,639,371$ & 53.7 & Nov 19 23:13:10 & 1626 & 1295 & 0.57 & 20.7 \\
\hline
\end{tabular}

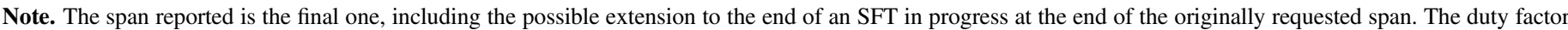

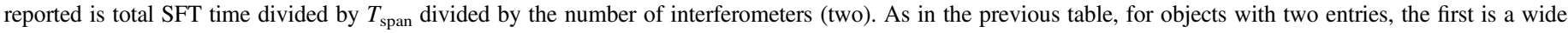

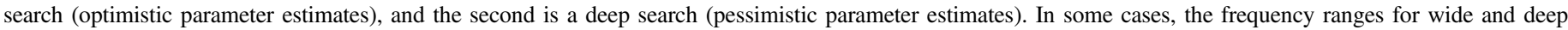
searches are nearly identical, but the ranges of spin-down parameters (described in the text) are not.

sensitivity would improve over the wide search by a factor of roughly $\sqrt{2}$.

The resulting targets and chosen parameters are shown in Table 1. We now briefly summarize each target and the provenance of the parameters used for it.

G1.9+0.3-Currently the youngest known SNR in the galaxy (Reynolds et al. 2008). Several arguments favor it being a Type Ia (Reynolds et al. 2008), which would leave no neutron star behind, but this is not definite, and the remnant's youth makes it an interesting target on the chance that it is not a Type Ia. We used the position of the center of the remnant from the discovery paper (Reich et al. 1984). At maximum kick velocity, any neutron star could have moved only a few arcseconds, which is not an issue for our searches. The age and distance shown are from the "rediscovery" paper (Reynolds et al. 2008), though the latter is a nominal galactic center distance.

G15.9+0.2-CCO discovered in Chandra data by Reynolds et al. (2006). We used the lower limit on age and the galactic center distance estimate from the same paper, though both quantities may be significantly greater (Klochkov et al. 2016).

G18.9-1.1-Position of the Chandra point source discovered by Tüllmann et al. (2010). Age and distance estimates are from the previous ROSAT and ASCA observations of Harrus et al. (2004).

G39.2-0.3-Also known as 3C 396. The PWN and embedded point source were found by Olbert et al. (2003) in Chandra data, the point source being localized to within $2^{\prime \prime}$ in spite of the PWN. Su et al. (2011) estimated the age and distance, the latter based on the tangent point of the spiral arm.

G65.7+1.2-Also known as DA 495. Arzoumanian et al. (2008) found the Chandra point source in the PWN. The quoted distance (Kothes et al. 2004) and minimum age (Kothes et al. 2008) are derived slightly inconsistently due to assumed distances to the galactic center. We did not attempt to resolve the inconsistency, though we did choose the distance from the former paper, since it uses the more commonly accepted galactic center distance. The latter paper (and others) also argue that the distance could be several times higher.

G93.3+6.9-Also known as DA 530. The position and age are from Jiang et al. (2007), and the distance estimate is from Foster \& Routledge (2003). Jiang et al. (2007) found no Chandra point source, but the X-ray intensity of the faint candidate PWN falls off on a scale of $6^{\prime \prime}$, which qualifies as a point source for our purposes.

G111.7-2.1-Also known as Cas A. The position of the CCO is from the Chandra "first light" observation (Tananbaum 1999), the distance is from Reed et al. (1995), and the age is from Fesen et al. (2006).

G189.1+3.0-Also known as IC 443. The position is that of the Chandra point source found by Olbert et al. (2001) embedded in the PWN. This object is often studied, with a wide range of distance and age estimates in the literature. We used Petre et al. (1988) for an optimistic age estimate. Our pessimistic age estimate is not quite the most extreme in the literature but rather a best fit for a pessimistic scenario from relatively recent modeling (Swartz et al. 2015). We did not use the most optimistic distance quoted but rather the assumed association with the I Gem cluster from Fesen \& Kirshner (1980), which is common in the literature.

G266.2-1.2-Also known as Vela Jr. The position is that of the CCO found by Pavlov et al. (2001). We used Iyudin et al. (1998) for the most optimistic age and distance estimates. The pessimistic age estimate is from Allen et al. (2015), which was published too recently for the previous paper in this series (Aasi et al. 2015). Allen et al. (2015) also discussed the possible association of several surrounding objects with the nearer concentration of the Vela Molecular Ridge at a spread of 

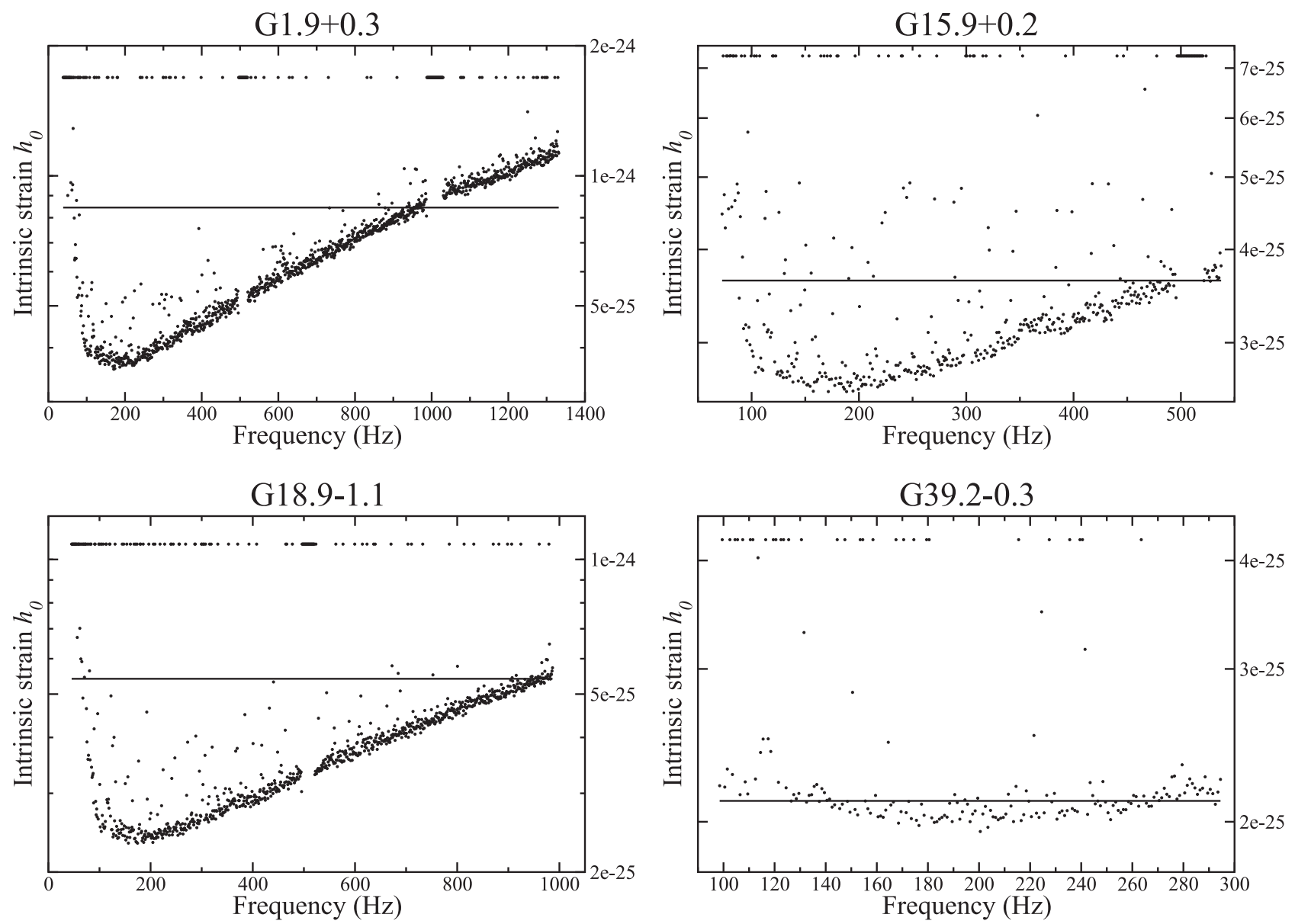

Figure 1. Direct observational 95\% confidence upper limits on intrinsic strain as a function of frequency in $1 \mathrm{~Hz}$ bands for four searches. The horizontal line indicates the indirect limit from energy conservation. Scattered points on a higher line indicate $1 \mathrm{~Hz}$ bands where no upper limit was set due to data quality issues. All figures trace a slightly distorted version of the noise curve, with G39.2-0.3 appearing flat because it covers only the bottom of the curve.

distances providing our pessimistic distance estimate (Liseau et al. 1992) and rendering the more pessimistic ones unlikely.

G291.0-0.1-Also known as MSH 11-62. The position and age are from the Chandra point-source discovery paper (Slane et al. 2012). The distance is from Moffett et al. (2001). The age and distance are derived in slightly inconsistent ways, but rather than attempt to repeat the calculations, we used the numbers quoted in the literature.

G330.2+1.0 - CCO discovered by Park et al. (2006) in Chandra data with subarcsecond position accuracy. We used a distance estimate from radio observations (McClure-Griffiths et al. 2001) and an age estimate from the X-ray spectrum (Park et al. 2009).

G347.3-0.5-Subarcsecond position obtained from archival Chandra data (Mignani et al. 2008), although the CCO had been identified in ASCA data earlier (Slane et al. 1999). We used the distance from Cassam-Chenaï et al. (2004) and the age from the proposed identification with a possible SN 393 (Wang et al. 1997). Although this identification may be problematic, given the inferred properties of such a supernova, other age estimates are comparable (Fesen et al. 2012).

G350.1-0.3-Position and distance estimates are from the discovery paper of the CCO candidate by Gaensler et al. (2008). The age is from Chandra observations (Lovchinsky et al. 2011).

G353.6-0.7-Most likely of several candidate CCOs identified by Halpern \& Gotthelf (2010). The age estimate (Tian et al. 2008) makes this CCO candidate the only one that is almost certainly too old for $r$-modes, although we still set upper limits on $r$-mode amplitude. The distance estimate is also from Tian et al. (2008). We used the first-observation position contained in the name of the candidate $\mathrm{CCO}$ rather than the slightly better Chandra position reported by Halpern \& Gotthelf (2010); the roughly $1^{\prime \prime}$ difference is not significant for the GW integration times used in this paper.

G354.4+0.0 - All parameters from the discovery paper (Roy $\&$ Pal 2013). No associated point source has been detected yet, but if the remnant's age is correct, any young neutron star should be within roughly $20^{\prime \prime}$ of the center (whose location we used for the $\mathrm{GW}$ search). Such a position error is not significant for the integration times used here.

Fomalhaut $b$ - Considered an extrasolar planet candidate since its discovery in a visible-light image (Kalas et al. 2008). Based on a lack of infrared detection, it has been proposed to be a serendipitous discovery of a nearby neutron star (Neuhäuser et al. 2015). Parameters are taken from Neuhäuser et al. (2015), with the maximum distance an attempt to balance the uncertainties in the scenarios discussed there. After this search was run, Poppenhaeger et al. (2017) searched for and did not find the object with Chandra. If the object is a neutron star, this somewhat reduces the possible distance and significantly increases the minimum age.

\subsection{Parameter Space}

After sky position, the key parameters for each search were the $\mathrm{GW}$ frequency band $\left(f_{\min }, f_{\max }\right)$ and time span of 

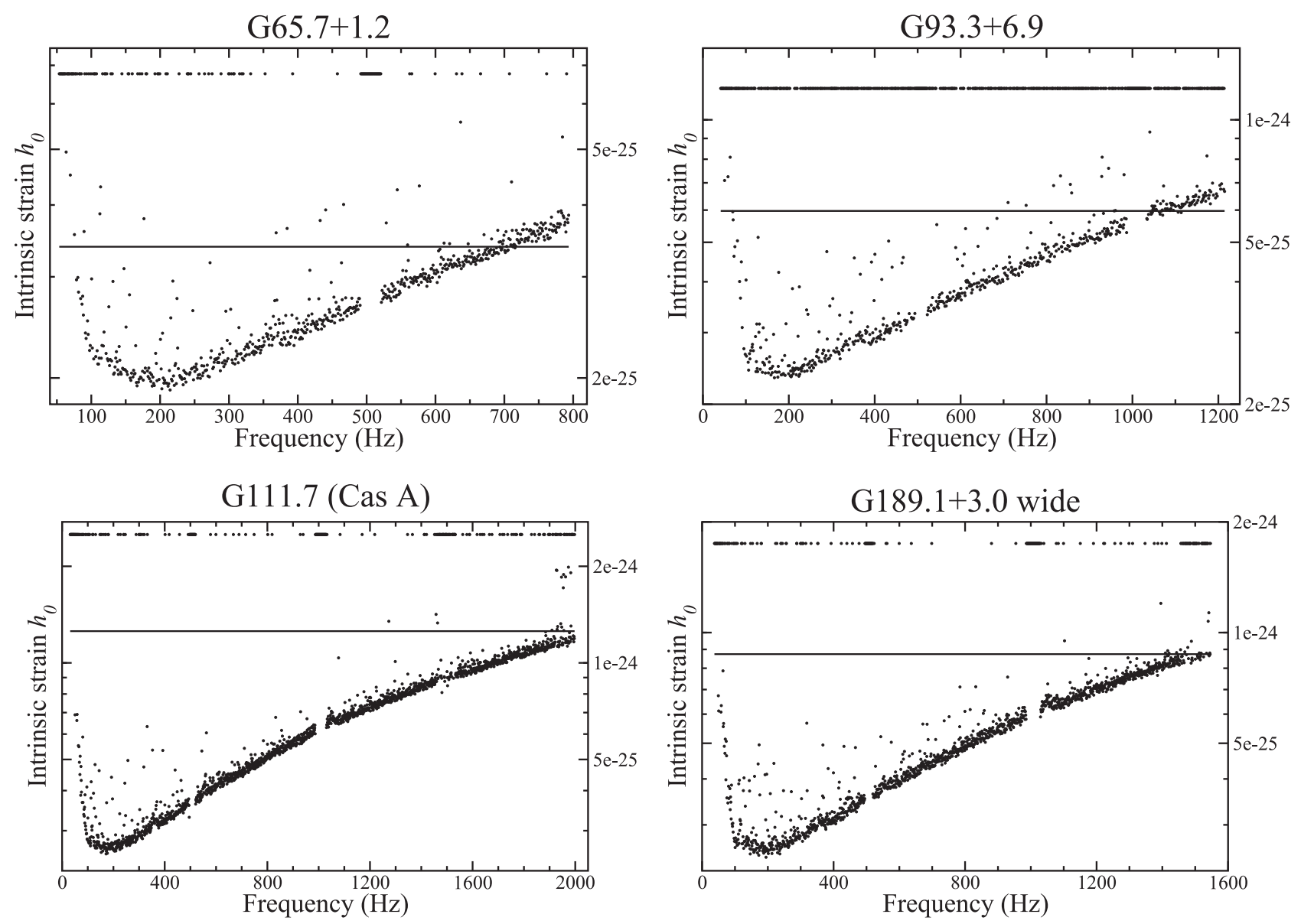

Figure 2. Same as Figure 1 for four more searches.

integration $T_{\text {span }}$. As in Aasi et al. (2015), these parameters were determined in an iterative process intended to produce a search more sensitive than $h_{0}^{\text {age }}$ over as wide a frequency band as possible for a fixed computational cost. Due to Doppler shifts and several features of the analysis, we capped the maximum frequency at $1998 \mathrm{~Hz}$ rather than the $2 \mathrm{kHz}$ in the SFTs. The cost, approximated as proportional to $a^{-1.1} f_{\max }^{2.2} T_{\text {span }}^{4}$, was kept comparable to that of Abadie et al. (2010) for most targets, but Cas A was allocated 10 times as many computational cycles due to its status as the youngest known neutron star in the galaxy. Due to some inaccuracy in the power-law fit used for computational cost as a function of the key parameters, the computational cost and sensitivity varied by up to $20 \%-30 \%$ from these goals. For a given frequency $f$, as in Abadie et al. (2010) and Aasi et al. (2015), we searched

$$
-\frac{f}{a} \leqslant \dot{f} \leqslant-\frac{1}{6} \frac{f}{a}
$$

and, for a given $\dot{f}$, we searched

$$
2 \frac{\dot{f}^{2}}{f} \leqslant \ddot{f} \leqslant 7 \frac{\dot{f}^{2}}{f} .
$$

These ranges and the computational cost fixed $f_{\min }, f_{\max }$, and $T_{\text {span }}$ for each search.

We then chose the start time of each search by the same method as Abadie et al. (2010) and Aasi et al. (2015), minimizing the harmonic mean of the strain noise power spectral density during the span over the frequency band $\left(f_{\min }, f_{\max }\right)$. Neglecting the small effect of the decl. of the target, this corresponds to maximizing the search sensitivity for a fixed $T_{\text {span }}$, which is roughly a fixed computational cost. Hence, the algorithm chose spans when both interferometers had good noise performance and little downtime, usually later in O1. The resulting search parameters are described in Table 2.

We applied the same consistency checks as in previous searches. For each search, we checked using the parameter space metric (Whitbeck 2006; Wette et al. 2008) that neglect of the third frequency derivative in Equation (1) did not significantly reduce $2 \mathcal{F}$, even in the worst case $(\mathrm{G} 1.9+0.3)$. We also checked that the position uncertainties of the targets also did not significantly reduce $2 \mathcal{F}$. A simple approximation (Whitbeck 2006) suggests that the sky resolution of these searches is an arcminute or two at $2 \mathrm{kHz}$ and a 10 day integration, and it scales inversely with $f_{\max }$ and $T_{\text {span }}$. We spotchecked this with injection studies and found it to be accurate. Given the integration times in Table 2, even the worst position uncertainty ( $20^{\prime \prime}$ for G354.4+0.0) is well within bounds for a single directed search. Finally, we checked that the standard $1800 \mathrm{~s}$ SFT duration did not diminish sensitivity to signals with $\dot{f}$ high enough that the frequency could move to another SFT frequency bin over the duration of the SFT. This effect was negligible, except for SNR G1.9+0.3, where it could reduce the sensitivity (raise the detectable $h_{0}$ ) by of order $10 \%$ at frequencies above $1 \mathrm{kHz}$. 

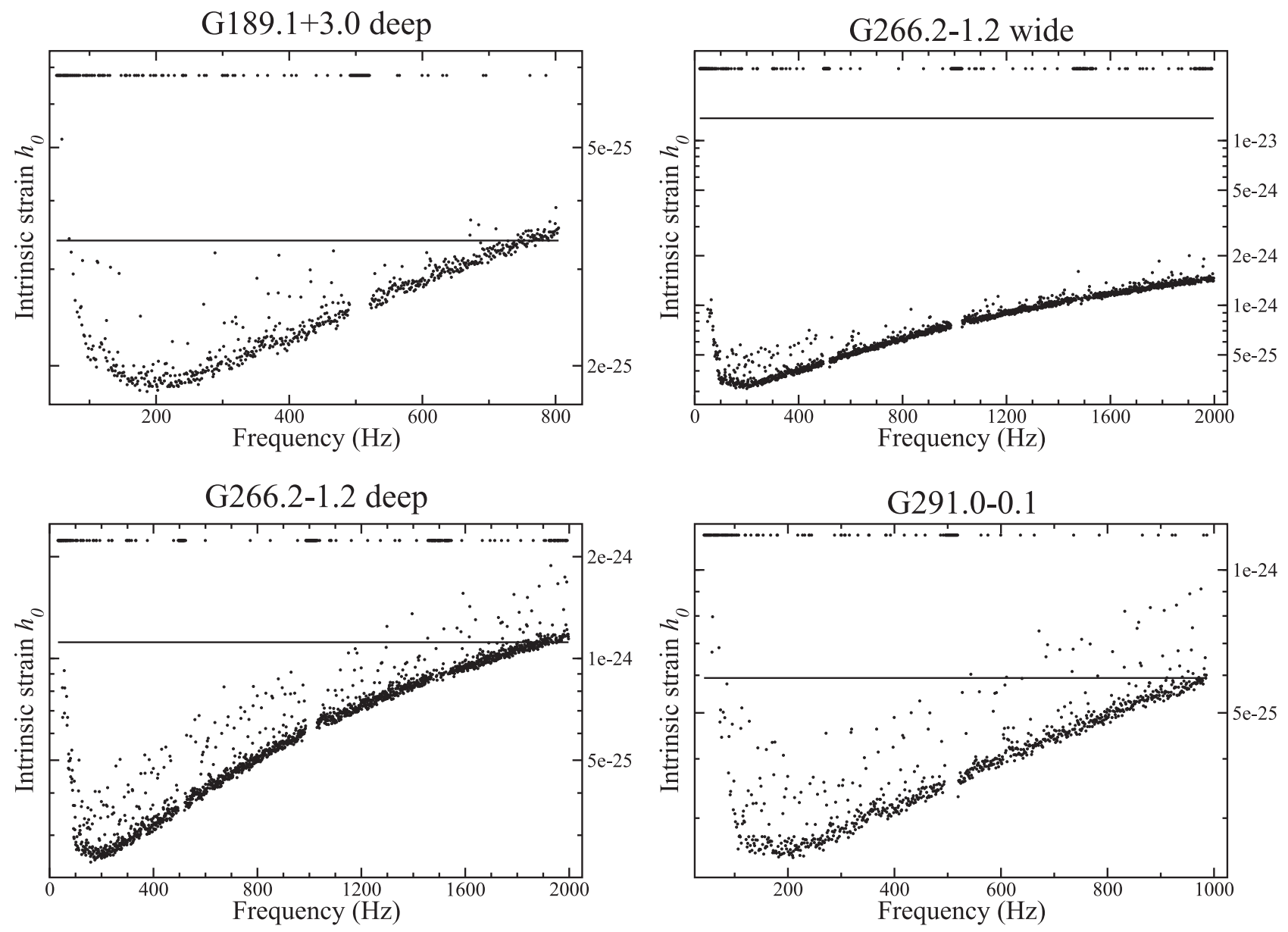

Figure 3. Same as Figure 1 for four more searches.

\subsection{Post-processing}

Each search recorded a list of candidates with high values of $2 \mathcal{F}$, which was then pared using two automated vetoes designed for instrumental artifacts, as used in Abadie et al. (2010) and Aasi et al. (2015). The "Fscan veto" used a normalized spectrogram formed from the SFTs to detect and veto spectral lines and nonstationary noise. Its implementation and parameters were the same as in Aasi et al. (2015), except that we fixed a bug in the old code whereby the Doppler shift due to the Earth's orbital motion was not applied. (This bug allowed more noise lines to pass the automated vetoes and require manual scrutiny but had a negligible effect on the falsedismissal rate.) The "interferometer consistency veto" ruled out candidates for which a single-interferometer $2 \mathcal{F}$ exceeded the two-interferometer $2 \mathcal{F}$ for the same event, indicating a disturbance present in only one interferometer. It also vetoed entire search jobs if the number of candidates vetoed was high enough. This veto was also applied in the same way as in Aasi et al. (2015), except that the threshold for vetoing an entire search job was $5 \%$ of the templates in that job. Unlike in previous papers in this series, we also vetoed a list of known instrumental spectral lines compiled from studies of the interferometers (Covas et al. 2018).

After these steps, including fixing the Doppler bug, the searches still had almost 2000 jobs containing nonvetoed outliers above the $95 \%$ confidence level for Gaussian noise. All of these jobs were examined by hand. As in Aasi et al. (2015), two plots were made and inspected for each job. (See Figure 1 of that paper for illustrative examples.) In the case of a real or injected signal, the first plot, of $2 \mathcal{F}$ versus frequency for all loud candidates in the job, would show a $\delta$-function-like spike even for very loud signals, as verified by studying hardware injections. The candidates generally showed broad bands of high noise, occupying a fraction of order unity of the search job frequency band, except for a handful that occupied a few percent of the search band. These few candidates, which were still of order 100 times broader than a real signal would be, were verified to be hardware-injected test signals detectable in the wrong sky location due to their huge amplitudes. The second plot for each search job containing candidates was a semilog histogram of loud candidates, which, on inspection, typically showed the tail of a $\chi^{2}(4)$ distribution with the wrong amplitude, indicative of a broadband disturbance in the noise spectrum. See Aasi et al. (2015) for examples and further details.

No candidates survived inspection of these plots; therefore, we conclude that no astrophysical signal was detected.

\section{Upper Limits}

Our method of setting upper limits was almost the same as in previous papers (Abadie et al. 2010; Aasi et al. 2015). In each $1 \mathrm{~Hz}$ band searched, we estimated the value of $h_{0}$ that would be detected $95 \%$ of the time by our search (assuming random variation of other signal parameters, such as the inclination of the star's rotation axis to the line of sight) at a louder value than the loudest $2 \mathcal{F}$ actually recorded by the search in that band. We 

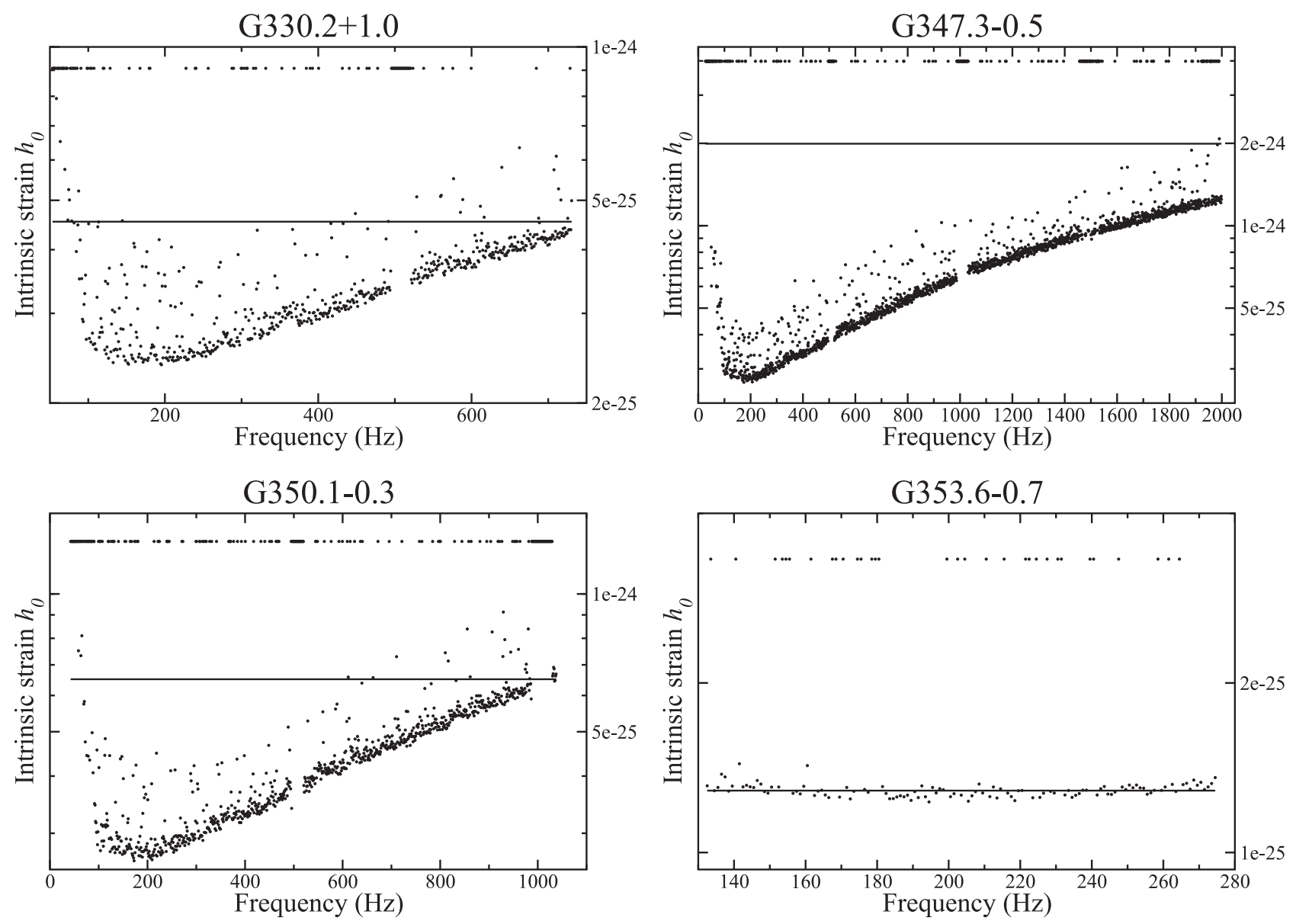

Figure 4. Same as Figure 1 for four more searches.

made an initial estimate from a semi-analytic integration of the expected $2 \mathcal{F}$ distribution. Then we injected simulated signals with different values of $h_{0}$ near this value to refine the location of the $95 \%$ confidence (5\% false-dismissal) threshold. We reduced the number of injections per band to 1000 (from 6000 in previous papers) due to the computational cost of setting upper limits on wider bands.

For each search, we pared the list of upper limits on $h_{0}$ versus frequency. We dropped bands where the injections indicated that the false-dismissal rate was more than 5\% and $\pm 1 \mathrm{~Hz}$ bands around the harmonics of the $60 \mathrm{~Hz}$ power mains up to $300 \mathrm{~Hz}$.

The resulting upper limits on $h_{0}$, in $1 \mathrm{~Hz}$ frequency bands, are plotted in Figures 1-5. Each curve has roughly the same shape as the amplitude spectral density of the strain noise. The line of dots near the top of each plot corresponds to bands where no upper limit was set. Some features, such as the "violin modes" of the interferometer test mass suspension (roughly $500 \mathrm{~Hz}$ and harmonics), are evident. The horizontal line in each plot is $h_{0}^{\text {age }}$, the strain the search was intended to beat. In some cases, the estimate of sensitivity made before performing the search was wrong by of order $10 \%$, so the upper limits (lower dots) do not always lie below the line.

Upper limits on $h_{0}$ can be converted to upper limits on fiducial neutron star ellipticity $\epsilon=\left|I_{x x}-I_{y y}\right| / I_{z z}$ (where $I_{a b}$ is the moment of inertia) using (e.g., Wette et al. 2008)

$$
\epsilon=9.5 \times 10^{-5}\left(\frac{h_{0}}{1.2 \times 10^{-24}}\right)\left(\frac{D}{1 \mathrm{kpc}}\right)\left(\frac{100 \mathrm{~Hz}}{f}\right)^{2} .
$$

This number assumes $I_{z z}=10^{45} \mathrm{~g} \mathrm{~cm}^{2}$. Uncertainties in the mass, radius, and neutron star equation of state make the conversion from $h_{0}$ to $\epsilon$ uncertain by a factor of 2 or more. This fiducial ellipticity can be converted to the true shape of the star (Johnson-McDaniel 2013) or other quantities (Owen 2010). We plot upper limits on $\epsilon$ for a selection of searches in the left panel of Figure 6. We do not plot the indirect limits on $\epsilon$ and $\alpha$ derived from $h_{0}^{\text {age }}$, since they are close to the direct upper limits on the scale of the plot. We do not plot the remaining searches because their upper limits are close to those of the searches plotted. The great differences between curves are mainly due to the distances to the sources; hence, Fomalhaut $b$ has the best upper limits, of order $10^{-9}$ at high frequencies.

Upper limits on $h_{0}$ can be converted to the common $r$-mode amplitude parameter $\alpha$ (Lindblom et al. 1998) via (Owen 2010)

$$
\alpha=0.028\left(\frac{h_{0}}{10^{-24}}\right)\left(\frac{100 \mathrm{~Hz}}{f}\right)^{3}\left(\frac{D}{1 \mathrm{kpc}}\right) .
$$



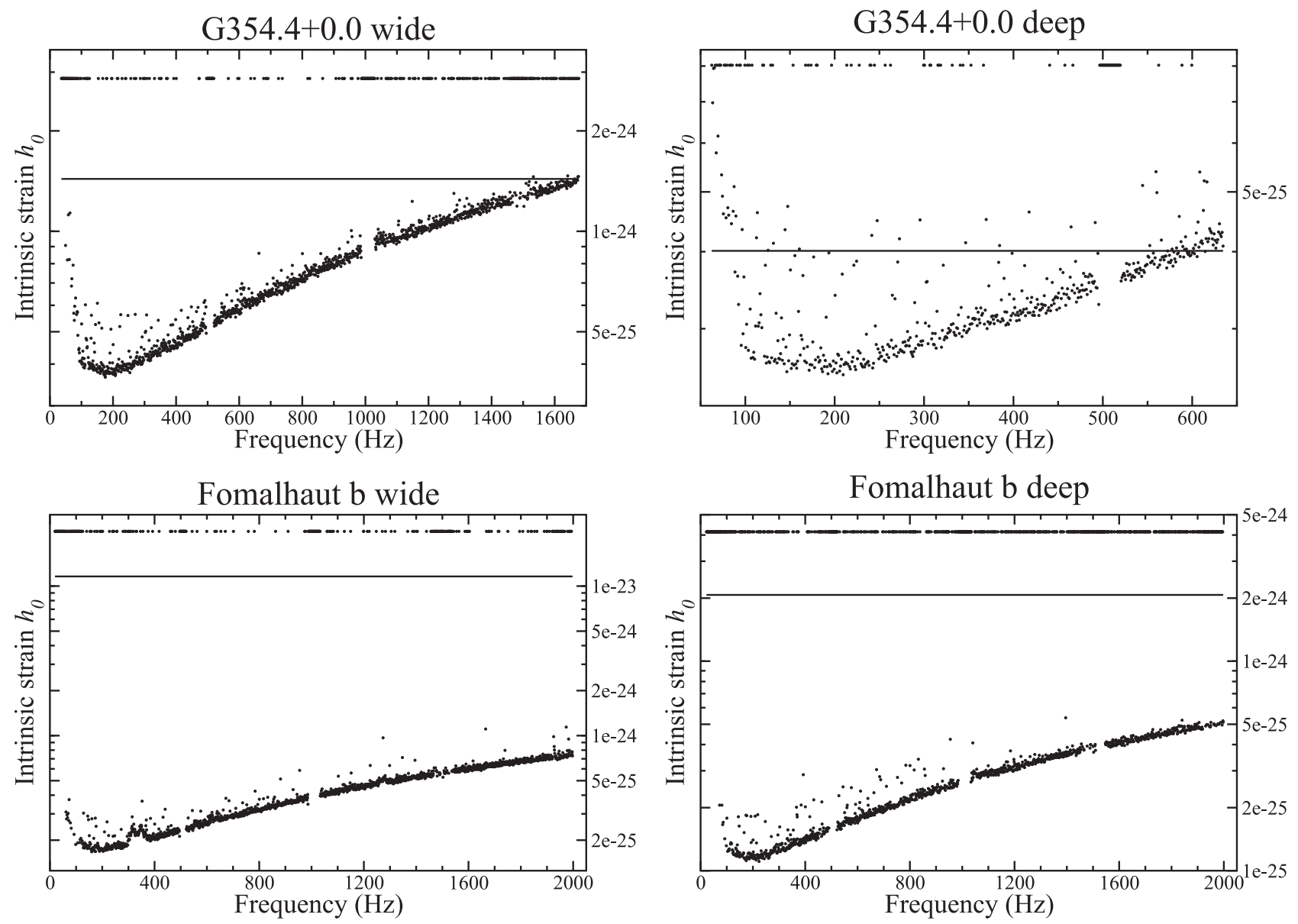

Figure 5. Same as Figure 1 for four more searches.
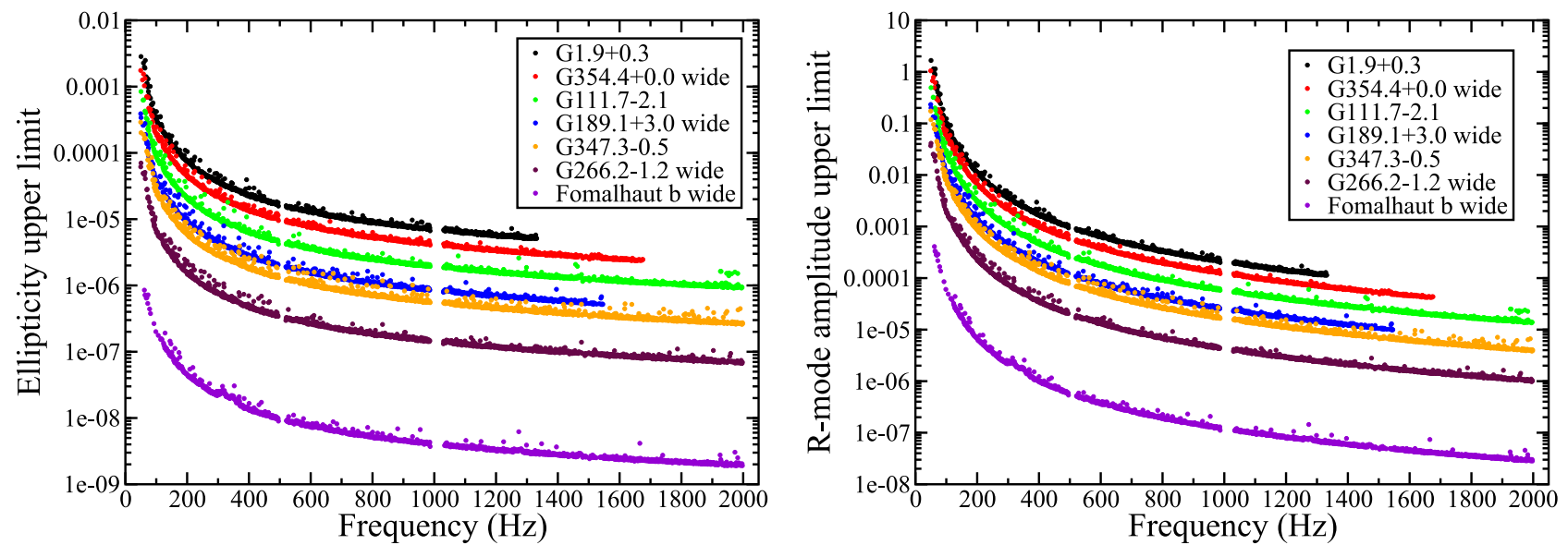

Figure 6. Upper limits on fiducial ellipticity (left panel) and $r$-mode amplitude (right panel) for a representative sample of searches.

This number assumes a fiducial set of stellar parameters described in Owen (2010) and is uncertain by a factor of up to about 3 , depending on the neutron star mass and equation of state. We plot upper limits on $\alpha$ for a selection of searches in the right panel of Figure 6. Again, the differences between curves are mainly due to the source distances. The best upper limits-apart from Fomalhaut b, which is almost certainly too old for active $r$-modes-are of order $10^{-6}$ at high frequencies for Vela Jr.

\section{Discussion}

These are the first directed searches of Advanced LIGO data using continuous wave analysis methods. These searches have improved on previous directed searches by covering wider parameter ranges and more targets and setting better upper limits on targets searched previously. Our upper limits on $h_{0}$ approach $2 \times 10^{-25}$ for many targets and $1 \times 10^{-25}$ for one target-about a factor of 3 improvement on Aasi et al. (2015), due mainly to the improvement in the detectors. Also, our 
upper limits beat the indirect limit $h_{0}^{\text {age }}$ over bands of $1-2 \mathrm{kHz}$ for more targets than were ever published before. (Searches for some of these targets in less sensitive S6 data for the purpose of testing code were described in an unpublished thesis; Idrisy 2015.) As with previous data runs, we improved on the sensitivity of all-sky wideband searches (Abbott et al. 2018a) but did not match the sensitivity of searches for known pulsars with full timing solutions (Abbott et al. 2017d). As before, the directed searches described here also have the caveats that there might be no neutron star present in some cases, any neutron star might be spinning too slowly to be detected, and a neutron star spinning at a detectable frequency might glitch. The latter phenomenon in a CCO is now an observation (Gotthelf \& Halpern 2018) rather than a surmise and would somewhat reduce the sensitivity of these searches (Ashton et al. 2017). Even with some longer integration times here, timing noise is not an issue unless these objects are orders of magnitude noisier than known pulsars (Ashton et al. 2015).

Most of our upper limits on $\epsilon$ and $\alpha$ are competitive with the largest numbers predicted by theory. The maximum $\epsilon$ for "mountains" supported by elastic stresses of normal neutron star matter is probably $10^{-5}-10^{-6}$ (Horowitz \& Kadau 2009; Johnson-McDaniel \& Owen 2013; Baiko \& Chugunov 2018), and for many of our searches, upper limits are in this region over hundreds of $\mathrm{Hz}$. The maximum $\alpha$ (nonlinear saturation amplitude) for $r$-modes is probably of order $10^{-3}$ (Bondarescu et al. 2009), and for many of our searches, upper limits beat this over hundreds of $\mathrm{Hz}$. Mountains supported by an internal magnetic field can produce $\epsilon$ of order $10^{-4}\left(B / 10^{15} \mathrm{G}\right)^{2}$, where $B$ is the poloidal part of the field (e.g., Ciolfi \& Rezzolla 2013). Since, unlike elastic mountains, magnetic mountains are likely to be within about an order of magnitude of this limit for a given internal field, depending on its configuration, our upper limits on $\epsilon$ translate into rough limits on the internal magnetic field if a neutron star is present and spinning rapidly enough to emit GWs in band.

More data from Advanced LIGO and Advanced Virgo are now available, with more live time and lower noise amplitude than before. The detectable values of intrinsic strain, ellipticity, and $r$-mode amplitude are proportional to the noise amplitude and the inverse square root of the live time. This makes more targets feasible for directed searches at greater sensitivity, increasing the chances of a detection of continuous GWs. Such searches will be done in the near future.

The authors gratefully acknowledge the support of the United States National Science Foundation (NSF) for the construction and operation of the LIGO Laboratory and Advanced LIGO, as well as the Science and Technology Facilities Council (STFC) of the United Kingdom, the MaxPlanck-Society (MPS), and the State of Niedersachsen/ Germany for support of the construction of Advanced LIGO and construction and operation of the GEO600 detector. Additional support for Advanced LIGO was provided by the Australian Research Council. The authors gratefully acknowledge the Italian Istituto Nazionale di Fisica Nucleare (INFN), the French Centre National de la Recherche Scientifique (CNRS), and the Foundation for Fundamental Research on Matter, supported by the Netherlands Organisation for Scientific Research, for the construction and operation of the Virgo detector and the creation and support of the EGO consortium. The authors also gratefully acknowledge research support from these agencies, as well as by the Council of Scientific and Industrial Research of India; the Department of Science and Technology, India; the Science \& Engineering Research Board (SERB), India; the Ministry of Human Resource Development, India; the Spanish Agencia Estatal de Investigación; the Vicepresidència i Conselleria d'Innovació Recerca i Turisme and the Conselleria d'Educació i Universitat del Govern de les Illes Balears; the Conselleria d'Educació Investigació Cultura i Esport de la Generalitat Valenciana; the National Science Centre of Poland; the Swiss National Science Foundation (SNSF); the Russian Foundation for Basic Research; the Russian Science Foundation; the European Commission; the European Regional Development Funds (ERDF); the Royal Society; the Scottish Funding Council; the Scottish Universities Physics Alliance; the Hungarian Scientific Research Fund (OTKA); the Lyon Institute of Origins (LIO), the Paris Île-de-France Region; the National Research, Development and Innovation Office Hungary (NKFIH); the National Research Foundation of Korea; Industry Canada and the Province of Ontario through the Ministry of Economic Development and Innovation; the Natural Science and Engineering Research Council Canada; the Canadian Institute for Advanced Research; the Brazilian Ministry of Science, Technology, Innovations, and Communications; the International Center for Theoretical Physics South American Institute for Fundamental Research (ICTP-SAIFR); the Research Grants Council of Hong Kong; the National Natural Science Foundation of China (NSFC); the Leverhulme Trust; the Research Corporation; the Ministry of Science and Technology (MOST), Taiwan; and the Kavli Foundation. The authors gratefully acknowledge the support of the NSF, STFC, MPS, INFN, CNRS, and State of Niedersachsen/Germany for provision of computational resources.

This paper has been assigned document No. LIGOP1800333. Data files for the figures are available at https:// dcc.ligo.org/P1800333/public.

Software: LALSuite (LIGO Scientific Collaboration 2018).

\section{References}

Aasi, J., Abadie, J., Abbott, B. P., et al. 2013, PhRvD, 88, 102002 Aasi, J., Abbott, B. P., Abbott, R., et al. 2015, ApJ, 813, 39 Abadie, J., Abbott, B. P., Abbott, R., et al. 2011, PhRvL, 107, 271102 Abadie, J., Abbott, B. P., Abbott, R., et al. 2010, ApJ, 722, 1504 Abbott, B. P., Abbott, R., Abbott, T. D., et al. 2016a, PhRvL, 116, 131103 Abbott, B. P., Abbott, R., Abbott, T. D., et al. 2016b, PhRvL, 116, 241103 Abbott, B. P., Abbott, R., Abbott, T. D., et al. 2016c, PhRvL, 116, 061102 Abbott, B. P., Abbott, R., Abbott, T. D., et al. 2017a, PhRvD, 95, 062003 Abbott, B. P., Abbott, R., Abbott, T. D., et al. 2017b, PhRvL, 118, 121102 Abbott, B. P., Abbott, R., Abbott, T. D., et al. 2017c, ApJ, 851, 71 Abbott, B. P., Abbott, R., Abbott, T. D., et al. 2017d, ApJ, 839, 12 Abbott, B. P., Abbott, R., Abbott, T. D., et al. 2017e, PhRvL, 118, 221101 Abbott, B. P., Abbott, R., Abbott, T. D., et al. 2017f, ApJL, 851, L35 Abbott, B. P., Abbott, R., Abbott, T. D., et al. 2017g, PhRvL, 119, 141101 Abbott, B. P., Abbott, R., Abbott, T. D., et al. 2017h, PhRvL, 119, 161101 Abbott, B. P., Abbott, R., Abbott, T. D., et al. 2017i, ApJL, 848, L12 Abbott, B. P., Abbott, R., Abbott, T. D., et al. 2017j, PhRvD, 95, 082005 Abbott, B. P., Abbott, R., Abbott, T. D., et al. 2018a, PhRvD, 97, 102003 Abbott, B. P., Abbott, R., Abbott, T. D., et al. 2018b, PhRvL, 121, 161101 Allen, G., Chow, K., DeLaney, T., et al. 2015, ApJ, 798, 82

Arzoumanian, Z., Safi-Harb, S., Landecker, T. L., Kothes, R., \& Camilo, F. 2008, ApJ, 687, 505

Ashton, G., Jones, D. I., \& Prix, R. 2015, PhRvD, 91, 062009 Ashton, G., Prix, R., \& Jones, D. I. 2017, PhRvD, 96, 063004 Baiko, D. A., \& Chugunov, A. I. 2018, MNRAS, 480, 5511

Bondarescu, R., Teukolsky, S. A., \& Wasserman, I. 2009, PhRvD, 79, 104003 Brady, P. R., Creighton, T., Cutler, C., \& Schutz, B. F. 1998, PhRvD, 57, 2101 Cassam-Chenaï, G., Decourchelle, A., Ballet, J., et al. 2004, A\&A, 427, 199 
Ciolfi, R., \& Rezzolla, L. 2013, MNRAS, 435, L43

Covas, P. B., Effler, A., Goetz, E., et al. 2018, PhRvD, 97, 082002

Cutler, C., \& Schutz, B. F. 2005, PhRvD, 72, 063006

De, S., Finstad, D., Lattimer, J. M., et al. 2018, PhRvL, 121, 091102

Fesen, R. A., Hammell, M. C., Morse, J., et al. 2006, ApJ, 645, 283

Fesen, R. A., \& Kirshner, R. P. 1980, ApJ, 242, 1023

Fesen, R. A., Kremer, R., Patnaude, D., \& Milisavljevic, D. 2012, AJ, 143, 27

Foster, T., \& Routledge, D. 2003, ApJ, 598, 1005

Gaensler, B. M., Tanna, A., Slane, P. O., et al. 2008, ApJL, 680, L37

Glampedakis, K., \& Gualtieri, L. 2018, in The Physics and Astrophysics of Neutron Stars, Astrophysics and Space Science Library, Vol. 457 (Cham: Springer), 673

Gotthelf, E. V., \& Halpern, J. P. 2018, ApJ, 866, 154

Green, D. A. 2014, BASI, 42, 47

Halpern, J. P., \& Gotthelf, E. V. 2010, ApJ, 710, 941

Harrus, I. M., Slane, P. O., Hughes, J. P., \& Plucinsky, P. P. 2004, ApJ, 603, 152

Horowitz, C. J., \& Kadau, K. 2009, PhRvL, 102, 191102

Idrisy, A. 2015, PhD thesis, The Pennsylvania State Univ.

Iyudin, A. F., Schönfelder, V., Bennett, K., et al. 1998, Natur, 396, 142

Jaranowski, P., Królak, A., \& Schutz, B. F. 1998, PhRvD, 58, 063001

Jiang, B., Chen, Y., \& Wang, Q. D. 2007, ApJ, 670, 1142

Johnson-McDaniel, N. K. 2013, PhRvD, 88, 044016

Johnson-McDaniel, N. K., \& Owen, B. J. 2013, PhRvD, 88, 044004

Kalas, P., Graham, J. R., Chiang, E., et al. 2008, Sci, 322, 1345

Klochkov, D., Suleimanov, V., Sasaki, M., \& Santangelo, A. 2016, A\&A, 592, L12

Kothes, R., Landecker, T. L., Reich, W., Safi-Harb, S., \& Arzoumanian, Z. 2008, ApJ, 687, 516

Kothes, R., Landecker, T. L., \& Wolleben, M. 2004, ApJ, 607, 855

LIGO Scientific Collaboration 2018, LIGO Algorithm Library-LALSuite, free software (GPL), doi:10.7935/GT1W-FZ16

Lindblom, L., Owen, B. J., \& Morsink, S. M. 1998, PhRvL, 80, 4843

Liseau, R., Lorenzetti, D., Nisini, B., Spinoglio, L., \& Moneti, A. 1992, A\&A, 265,577

Lovchinsky, I., Slane, P., Gaensler, B. M., et al. 2011, ApJ, 731, 70

McClure-Griffiths, N. M., Green, A. J., Dickey, J. M., et al. 2001, ApJ, 551,394
Mignani, R. P., Zaggia, S., de Luca, A., et al. 2008, A\&A, 484, 457

Moffett, D., Gaensler, B., \& Green, A. 2001, in AIP Conf. Proc. 565, Young Supernova Remnants: Eleventh Astrophysics Conf., ed. S. S. Holt \& U. Hwang (Melville, NY: AIP), 333

Neuhäuser, R., Hohle, M. M., Ginski, C., et al. 2015, MNRAS, 448, 376

Olbert, C. M., Clearfield, C. R., Williams, N. E., Keohane, J. W., \& Frail, D. A. 2001, ApJL, 554, L205

Olbert, C. M., Keohane, J. W., Arnaud, K. A., et al. 2003, ApJL, 592, L45

Owen, B. J. 1996, PhRvD, 53, 6749

Owen, B. J. 2009, arXiv:0903.2603

Owen, B. J. 2010, PhRvD, 82, 104002

Park, S., Kargaltsev, O., Pavlov, G. G., et al. 2009, ApJ, 695, 431

Park, S., Mori, K., Kargaltsev, O., et al. 2006, ApJL, 653, L37

Pavlov, G. G., Sanwal, D., Kizıltan, B., \& Garmire, G. P. 2001, ApJL, 559, L131

Petre, R., Szymkowiak, A. E., Seward, F. D., \& Willingale, R. 1988, ApJ, 335,215

Poppenhaeger, K., Auchettl, K., \& Wolk, S. J. 2017, MNRAS, 468, 4018

Reed, J. E., Hester, J. J., Fabian, A. C., \& Winkler, P. F. 1995, ApJ, 440, 706

Reich, W., Fuerst, E., Haslam, C. G. T., Steffen, P., \& Reif, K. 1984, A\&AS, 58, 197

Reynolds, S. P., Borkowski, K. J., Green, D. A., et al. 2008, ApJL, 680, L41

Reynolds, S. P., Borkowski, K. J., Hwang, U., et al. 2006, ApJL, 652, L45

Roy, S., \& Pal, S. 2013, ApJ, 774, 150

Slane, P., Gaensler, B. M., Dame, T. M., et al. 1999, ApJ, 525, 357

Slane, P., Hughes, J. P., Temim, T., et al. 2012, ApJ, 749, 131

Su, Y., Chen, Y., Yang, J., et al. 2011, ApJ, 727, 43

Sun, L., Melatos, A., Lasky, P. D., Chung, C. T. Y., \& Darman, N. S. 2016 PhRvD, 94, 082004

Swartz, D. A., Pavlov, G. G., Clarke, T., et al. 2015, ApJ, 808, 84

Tananbaum, H. 1999, IAUC, 7246, 1

Tian, W. W., Leahy, D. A., Haverkorn, M., \& Jiang, B. 2008, ApJL, 679, L85

Tüllmann, R., Plucinsky, P. P., Gaetz, T. J., et al. 2010, ApJ, 720, 848

Wang, Z. R., Qu, Q., \& Chen, Y. 1997, A\&A, 318, L59

Wette, K., Owen, B. J., Allen, B., et al. 2008, CQGra, 25, 235011

Whitbeck, D. M. 2006, PhD thesis, Pennsylvania State Univ.

Zhu, S. J., Papa, M. A., Eggenstein, H.-B., et al. 2016, PhRvD, 94, 082008 